Department of Mathematics

Scientific Computing

The Institute of Technology

Linköping University

\title{
Weak and Strong Wall Boundary Procedures and Convergence to Steady-State of the Navier-Stokes Equations
}

Jan Nordström, Sofia Eriksson and Peter Eliasson 
Series: LiTH-MAT-R; 15

ISRN: LiTH-MAT-R--2011/15--SE

Publishing year: 2011

(c) The Author(s) 
Series: LiTH-MAT-R; 15

ISRN: LiTH-MAT-R--2011/15--SE

Publishing year: 2011

(c) The Author(s) 


\title{
Weak and Strong Wall Boundary Procedures and Convergence to Steady-State of the Navier-Stokes Equations
}

\author{
Jan Nordström $^{\mathrm{a}, *}$, Sofia Eriksson ${ }^{\mathrm{b}}$, Peter Eliasson ${ }^{\mathrm{c}}$ \\ a Department of Mathematics, Scientific Computing, University of Linköping, \\ SE-581 83 Linköping, Sweden \\ ${ }^{\mathrm{b}}$ Department of Information Technology, Scientific Computing, Uppsala \\ University, SE-751 05 Uppsala, Sweden \\ ${ }^{\mathrm{c}}$ Department of Aeronautics and Systems Integration, FOI, The Swedish Defence \\ Research Agency, SE-16490 Stockholm, Sweden
}

\begin{abstract}
We study the influence of different implementations of no-slip solid wall boundary conditions on the convergence to steady-state of the Navier-Stokes equations. The various approaches are investigated using the energy method and an eigenvalue analysis. It is shown that the weak implementation is superior and enhances the convergence to steady-state for coarse meshes. It is also demonstrated that all the stable approaches produce the same convergence rate as the mesh size goes to zero. The numerical results obtained by using a fully nonlinear finite volume solver support the theoretical findings from the linear analysis.
\end{abstract}

Key words: Navier-Stokes, steady-state, boundary conditions, convergence, summation-by-parts

\section{Introduction}

The formulations of the solid wall boundary conditions for the Navier-Stokes equations and the related slip condition for the Euler equations are well known. Less well known is the relation between these two formulations with a weak implementation. One of the more striking features is the fact that with a weak implementation of the boundary condition, the velocity at the wall becomes

* Corresponding author, Email adress: Jan.Nordstrom@liu.se 
zero only for very fine meshes. It has also been shown in $[8,2,48,3]$ that the Navier-Stokes solution converge to the Euler solution for coarse meshes.

As an example, consider Figures 1 and 2 where a no-slip velocity is imposed through weak and strong boundary procedures. (We reserve the term boundary condition for the continuous problem and use boundary procedure when we refer to the numerical implementation of the boundary condition). The velocity field is illustrated on a coarse and fine grid. There is a small velocity component on the wall boundary for the coarse mesh with the weak implementation. The wall velocity is reduced as the grid is refined. Away from the wall there are small differences between the solutions.

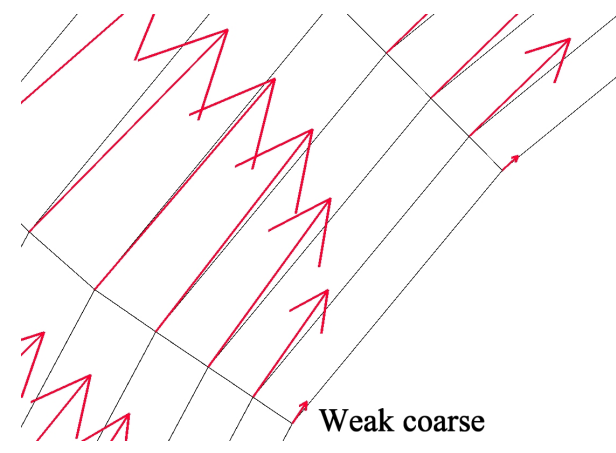

(a)

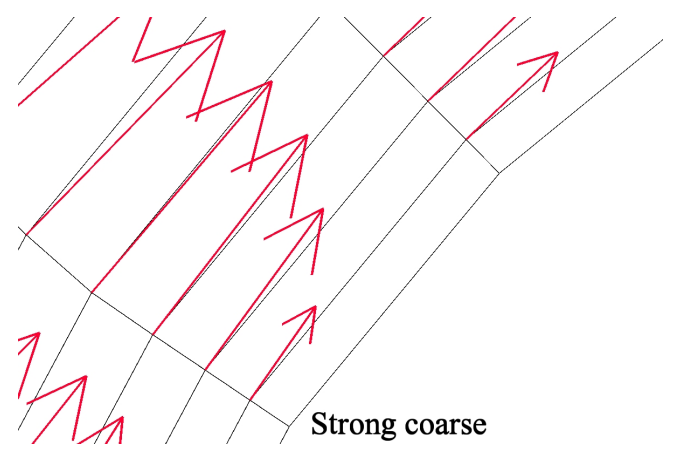

(b)

Fig. 1. The velocity field close to the solid wall for weak and strong implementation. Coarse mesh results, notice the non-zero slip velocity at the wall for the weak case.

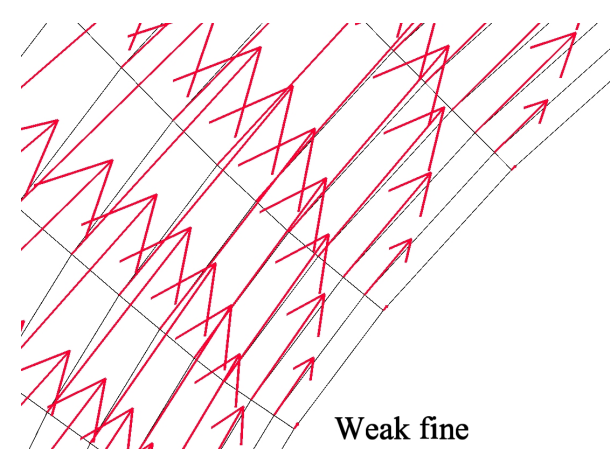

(a)

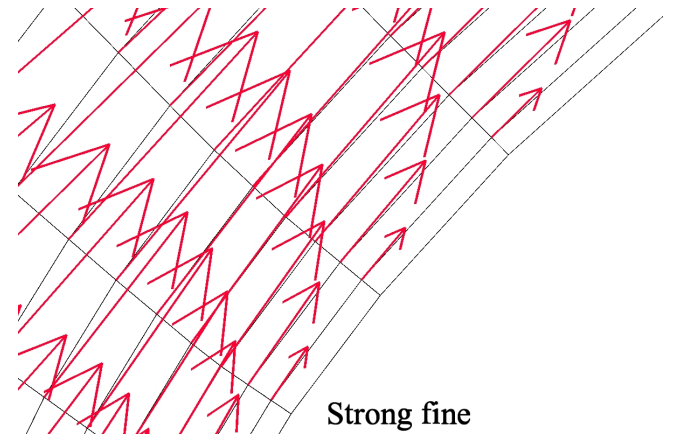

(b)

Fig. 2. The velocity field close to the solid wall for weak and strong implementation. Fine mesh results, notice the almost zero slip velocity at the wall for the weak case.

The main reason to use weak boundary procedures stems from the fact that together with summation-by-parts operators they lead to provable stable schemes. For application of this technique to finite difference methods, node-centered finite volume methods, spectral domain methods and various hybrid methods see $[25,42,4,30,31,36,44,48,20,39,6,22,13,24],[32,47,45,46,14,41],[18,16,19,7]$ and $[33,34,15,37,5]$ respectively. In this paper we will consider a new effect of using 
weak boundary procedures, namely that it in many cases (all that we tried) speeds up the convergence to steady-state.

Most of the work on convergence acceleration to steady-state is done for the discrete problem. Techniques such as local time-stepping, multigrid, residual smoothing, etc are used to enhance the convergence to steady-state, see for example $[23,28,49]$. In the majority of the investigations, the discrete problem including boundary conditions, is formulated first, and the numerical convergence acceleration technique is more or less independently added on afterwards. We will not consider this type of numerical convergence acceleration techniques but rather focus on more fundamental aspects related directly to the governing equations and the numerical scheme.

We will consider the basic requirement for convergence to steady-state, namely the position of the eigenvalues (the spectrum) of the Initial Boundary Value Problem (IBVP). The eigenvalues of the corresponding semi-discrete Initial Value Problem (IVP) problem obtained by using the method of lines, see [40], are equally important and will also be discussed. We start by formulating the relevant IBVP and the data requirements for the existence of a steadystate solution. Next we make sure that we have a well posed procedure (such that it is possible to reach the steady-state) and finally quantify the speed of convergence using the utmost right lying eigenvalue in the spectrum. That is repeated with minor technical modifications (such as replacing the concept well-posedness by stability, investigating numerical eigenvalues, etc.) for the IVP.

Next we apply the general theory to the specific case of solid wall boundary conditions. We continue the work in [10] and to some extent follow the procedure outlined in $[12,29]$ for the analysis of far field boundary conditions. Our basic theoretical tools will be the classical ones, namely the energy method, the Laplace transform technique and the matrix exponential. Our basic computational tool is a node vertex edge based flow solver for unstructured grids, the Edge code (see [9,11]). Edge has all the standard numerical convergence acceleration techniques described above, but as mentioned earlier, that technique will not be considered.

The paper is organized as follows. In section 2 we formulate the steady-state problem. Next, in section 3, the complete steady-state analysis for general IBVP's is presented. In section 4 we present the corresponding IVP. The corresponding steady-state analysis for the semi-discrete problem is displayed in section 5. The complete theory is applied to the particular problem with solid wall boundary conditions for the Navier-Stokes equations in section 6 , and the problem is simplified and analyzed in detail in section 7 . Numerical experiments supporting the theoretical analysis will be presented in section 8 and conclusions are drawn in section 9 . 


\section{The formulation of the IBVP for steady-state calculations}

Consider the following time-dependent one-dimensional model problem.

$$
\begin{aligned}
& u_{t}+\mathcal{A} u=F(x, t), \quad x \geq 0, \quad t \geq 0 \\
& L u=g(t), \quad x=0, \quad t \geq 0 \\
& u=f(x), \quad x \geq 0, \quad t=0,
\end{aligned}
$$

where $u(x, t)=\left(u^{1}, u^{2}, . ., u^{m}\right)^{T}$ is the solution vector with $m$ components, and $\mathcal{A}=\mathcal{A}(\partial / \partial x)$ is the differential operator. The forcing function $F$, the boundary data $g$ and the initial function $f$ are the data of the problem. For simplicity we disregard the influence of the right boundary.

Assume now that we want to use (1) and compute a steady solution $v$ that satisfies

$$
\begin{aligned}
\mathcal{A} v & =\tilde{F}(x), & & x \geq 0 \\
L v & =\tilde{g}, & & x=0 .
\end{aligned}
$$

The difference problem that describes the possible convergence to steady-state is obtained by subtracting (2) from (1). The deviation $e=u-v$ from steadystate satisfies

$$
\begin{aligned}
& e_{t}+\mathcal{A} e=d F(x, t), \quad x \geq 0, \quad t \geq 0 \\
& L e=d g(t), \quad x=0, \quad t \geq 0 \\
& e=d f(x), \quad x \geq 0, \quad t=0,
\end{aligned}
$$

where $d F(x, t)=F-\tilde{F}, d g(t)=g-\tilde{g}$ and $d f(x)=f-v$. Note that we have used the fact that $v_{t}=0$ to arrive at (3).

For a steady-state solution to exist, no time-dependent data are allowed. This means that $d F$ and $d g$ must vanish as $t \rightarrow \infty$. Furthermore, we cannot expect to be able to guess the initial condition that makes $d f=0$. The task is therefore to develop techniques for the IBVP (3) with zero forcing function, zero boundary data and non-zero initial function such that $e \rightarrow 0$ as $t \rightarrow \infty$.

For later reference, the final version of the steady-state problem under investigation in this paper is

$$
\begin{array}{rlrl}
e_{t}+\mathcal{A} e=0, & & x \geq 0, & t \geq 0 \\
L e=0, & x=0, & t \geq 0 \\
e=f, & x \geq 0, & t=0 .
\end{array}
$$

Our ambition is to reduce $e$ from its initial value $f$ and reach zero fast. 


\section{Convergence to steady-state of solutions to the IBVP}

We discuss the requirements for obtaining steady-state solutions $(e=0)$ in (4). In almost all practical cases, the spatial operator $\mathcal{A}$ is given. The speed at which $e \rightarrow 0$, the convergence rate to steady-state, can therefore only be manipulated by the boundary condition $L e=0$. Different choices of the boundary operator $L$ leads to different convergence rates.

\subsection{The Laplace-transform method for obtaining the convergence rate}

The convergence rate will be obtained by using the so called Laplace-transform technique, see $[12,29,17]$. Assume that a suitable boundary operator $L$ has been determined. The Laplace transformed version of (4) is

$$
\begin{aligned}
(s I+\mathcal{A}) \hat{e} & =f, \quad x \geq 0 \\
L \hat{e} & =0, \quad x=0
\end{aligned}
$$

where $s=\eta+i \xi$ is the dual variable to time and

$$
\hat{e}(x, s)=\mathcal{L} e=\int_{0}^{\infty} e(x, t) \exp (-s t) d t .
$$

The integral in (6) is well defined if the time-growth of the solution $e$ in (4) is bounded, i.e. if (4) is well posed and $\eta$ is sufficiently large and positive.

To solve (5) we make the ansatz $\hat{e}_{h}=\psi \exp (\kappa x)$ for the homogeneous solution. The particular solution $\hat{e}_{p}$ which depends on the initial data $f$ is assumed known. That leads to a generalized eigenvalue problem for $\kappa(s)$ of the form

$$
(s I+\mathcal{A}(\kappa)) \psi=0, \quad|s I+\mathcal{A}(\kappa)|=0 .
$$

The first equation in (7) has a non-trivial solution $\psi \neq 0$ if and only if there are $\kappa$ such that the second relation is satisfied. $\mathcal{A}(\kappa)$ is a polynomial in $\kappa$ with matrix coefficients. As an example, $\mathcal{A}=A \partial / \partial x+B \partial^{2} / \partial x^{2}$ leads to $\mathcal{A}(\kappa)=A \kappa+B \kappa^{2}$ where $A$ and $B$ are matrices. Note that $\psi=\psi(\kappa(s), s)$.

Assuming that there are no multiple generalized eigenvalues $\kappa$, the general homogeneous solution is

$$
\hat{e}_{h}=\sum_{i} \sigma_{i} \psi_{i} \exp \left(\kappa_{i} x\right) .=\Psi \bar{X}(x) \sigma
$$

where $\bar{X}(x)=\operatorname{diag}\left[e^{\kappa_{1} x}, e^{\kappa_{2} x}, \ldots\right], \Psi=\left[\psi_{1}, \psi_{2}, \ldots\right]$ and $\sigma=\left[\sigma_{1}, \sigma_{2}, \ldots\right]^{T}$. The coefficients $\sigma_{i}$ will be determined by the boundary conditions. The total solution is given by $\hat{e}=\hat{e}_{h}+\hat{e}_{p}$. The boundary conditions in (5) lead to $L \hat{e}_{h}=\hat{g}$ 
where $\hat{g}=-L \hat{e}_{p}$. By using (8), the final equation for the coefficients $\sigma_{i}$ becomes

$$
E(s) \sigma=\hat{g}, \quad E(s)=L \Psi \bar{X}(0) .
$$

$E(s)$ is a matrix with the structure given by the boundary operator $L$, the eigenvectors $\psi_{i}$ and the generalized eigenvalues $\kappa_{i}$. The right-hand side $\hat{g}$ is known and depend on the particular solution and it's gradients on the boundary.

A unique solution $\sigma$ of (9) is obtained if $E$ is non-singular. With a non-singular $E$, i.e. with $s$ such that $\eta>\eta^{*}$ where all the possible singularities (or eigenvalues) in $E$ lies to the left in the complex plane, we can solve for $\sigma$ and formally transform back to time domain by

$$
e(x, t)=\mathcal{L}^{-1} \hat{e}=\exp \left(\eta^{*} t\right)\left(\frac{1}{2 \pi} \int_{-\infty}^{+\infty} \hat{e}\left(x, \eta^{*}+i \xi\right) \exp (i \xi t) d \xi\right)
$$

Convergence to steady-state is obtained if $\eta^{*}<0$. The way to increase the convergence rate $\left(\eta^{*}\right)$ to steady-state is to choose the boundary operator $L$ such that $\eta^{*}$ lies as far as possible to the left in the complex plane. For later reference we denote $\eta^{*}=$ the continuos decay rate.

Remark: If there are multiple roots $\kappa$ to the first equation in (7), the ansatz $\hat{e}_{h}=\psi \exp (\kappa x)$ must be reformulated. Instead of a constant vector, $\psi$ is now of the form $\psi=\psi_{0}+x \psi_{1} \ldots+x^{n} \psi_{n}$, where $n+1$ is the multiplicity of the root $\kappa$. That complicates the derivation above technically, but in principle it remains the same. Also the conclusion that we have convergence to steadystate for $\eta^{*}<0$ remains the same. For more details in the multiple root case, see $[12,29,17]$.

\subsection{The energy method for deriving boundary conditions}

In the previous section, the boundary operator was assumed given. To derive suitable boundary operators $L$ such that $e \rightarrow 0$ fast as $t \rightarrow \infty$ we use the energy method. By choosing dissipative boundary operators $L$ we hope to push the spectrum (the utmost right lying eigenvalues) as far left as possible in the left half plane. Multiply (4) with $e^{T}$, add the transpose of the equation, and integrate over the domain. That leads to

$$
\|e\|_{t}^{2}=-\int_{0}^{\infty} e^{T}\left(\mathcal{A}+\mathcal{A}^{T}\right) e d x=B T\left(e, e_{x}\right)_{x=0}-\int_{0}^{\infty} R\left(e, e_{x}\right) d x .
$$

For (11) to be well posed, the right-hand side (RHS) in (11) must be bounded by const. $\|e\|^{2}$, see [17] for more details on well-posedness.

Remark: In (11) we have assumed that the operator $\mathcal{A}$ may include sec- 
ond derivatives (integration-by-parts yield first derivatives). All important hyperbolic (Euler, Maxwells, wave equations) and parabolic (heat, stress equations) as well as incompletely parabolic problems (Navier-Stokes equations) are thereby included.

For a fast convergence to steady-state (a negative $\eta^{*}$ with large magnitude), the RHS in (11) should be made as negative as possible. In almost all practical cases, the spatial operator $\mathcal{A}$ is given and hence also the original form of the RHS. There is no possibility to modify the volume term $R$ (which must be negative semi-definite). The rate at which the norm is decreasing (or increasing) can only be manipulated by the boundary condition $L e=0$. Different choices of $L$ leads to different sizes and signs of $B T\left(e, e_{x}\right)_{x=0}$ and different convergence rates.

Remark: No direct method for constructing boundary conditions that lead to fast convergence to steady-state exist. In this paper we assume that forcing the energy to decay fast, will lead to fast convergence to steady-state.

\section{The formulation of the IVP for steady-state calculations}

We discretize the IBVP (4) in space and leave time continuous, i.e. use the method of lines [40]. The focus is on the matrix properties of the resulting system of ordinary differential equations. The semi-discrete version of (4) can formally be written

$$
\begin{aligned}
\mathbf{e}_{t}+\bar{A} \mathbf{e} & =0, \quad t \geq 0 \\
\mathbf{e} & =f, \quad t=0 .
\end{aligned}
$$

where $\mathbf{e}=\left(e_{0}, e_{1}, . ., e_{N}\right)^{T}, e_{j}=\left(e^{1}, e^{2}, . ., e^{m}\right)_{j}^{T}$ is the discrete version of the deviation $e$ from steady-state. $\bar{A}=A-\Sigma$ is a modified version of $A$, where $A$ is the $(N+1) m \times(N+1) m$ discretization matrix approximating the operator $\mathcal{A}$ and $N+1$ is the number of grid points. When the scheme is adjusted to include the numerical treatment of the boundary conditions $(\Sigma)$ we obtain $\bar{A}$.

There are two distinctly different ways to prescribe boundary conditions for solvers where the unknowns are located on the boundary. One can use a weak or a strong boundary procedure. In a weak boundary procedure, the quantities at the boundaries, even though they are known, are updated in time. The boundary value typically deviates slightly from the prescribed value but the deviation is reduced as the grid is refined. With a strong boundary procedure, on the other hand, the specified boundary value is injected into the dependent variable on the boundary. The boundary quantity is no longer an unknown, and there is hence no need for an update. For more details on various boundary procedures, see [26]. 
In the case of a weak boundary procedure we have $\bar{A}=A-\Sigma$ where the matrix $A$ correspond to the internal discretisation (often on so called summation-byparts (SBP) form). The incorporation of the continuous boundary conditions $L e=0$ is done weakly by using the penalty term $\Sigma \mathbf{e}$. The form of the penalty matrix $\Sigma$ depend on the boundary operator $L$, i.e. $\Sigma=\Sigma(L)$. Roughly speaking, the penalty term must be accurate and lead to stability. For more details on SBP operators and penalty methods, see the references in the introduction. We will also go through it in detail below.

The way to incorporate the continuous boundary conditions using a strong implementation is not clearly separable from the internal discretisation (as is the weak boundary procedure). It essentially amounts to modifying the internal operator $A$ directly in the boundary region and turn it into $\bar{A}$. To facilitate the comparison with weak boundary conditions we reformulate the strong implementation such that it directly mimics the weak one. That means that we add and subtract terms to obtain the matrix relation $\bar{A}=A-\Sigma$ where $A$ is the same for both weak and strong boundary procedures.

\section{Convergence to steady-state of solutions to the IVP}

We discuss the requirements for obtaining steady-state solutions $\mathbf{e}=0$ in (12). The sign and size of the real part of the eigenvalues to $\bar{A}$ is the crucial factor. The eigenvalues of course depend on the discretisation of the spatial operator as well as on the boundary procedure. We will focus on the influence of the boundary procedure and keep the discretisation of the spatial operator fixed.

\subsection{The matrix exponential method for obtaining the convergence rate}

Once we have the IVP on the form (12) we can write down the solution as

$$
\mathbf{e}=\exp (-\bar{A} t) f, \quad t \geq 0
$$

using the definition of the matrix exponential. Next we assume that $\bar{A}$ is diagonalizable (the algebraic multiplicity of the eigenvalues $\lambda_{i}$ is equal to the geometric multiplicity) such that $\bar{A}=X \Lambda X^{-1}$ where $\Lambda=\operatorname{diag}\left(\lambda_{i}\right)$. That leads to

$$
\mathbf{e}=X \exp (-\Lambda t) X^{-1} f, \quad t \geq 0
$$

and convergence to steady-state if all $\Re\left(\lambda_{i}\right)>0$. The way to increase the convergence rate $\left(\min _{i} \Re\left(\lambda_{i}\right)\right)$ to steady-state is to choose a boundary procedure such that $\min _{i} \Re\left(\lambda_{i}\right)$ lies as far as possible to the right in the complex plane. Note that the sign convention used here is such that $\min _{i} \Re\left(\lambda_{i}\right) \rightarrow-\eta^{*}$ as the mesh is refined. 
Remark: If there are multiple roots $\lambda_{i}$ such that $\bar{A}$ is not diagonalizable (the algebraic multiplicity of the eigenvalues $\lambda_{i}$ is larger than the geometric multiplicity), then we can use the Jordan decomposition of $\bar{A}$. The derivation above remains almost the same and the conclusion that we have convergence to steady-state for $\min _{i} \Re\left(\lambda_{i}\right)>0$ remains exactly the same. For more details in the multiple root case, see $[12,29,17]$.

\subsection{The energy method for deriving boundary procedures}

To derive a suitable discretization, i.e. a matrix $\bar{A}$ with eigenvalues such that $\mathbf{e} \rightarrow 0$ as $t \rightarrow \infty$ as was discussed above, we use the energy method. Multiply (12) with $\mathbf{e}^{T} \bar{P}$ from the left, where $\bar{P} \equiv\left(P \otimes I_{m}\right)$. $I_{m}$ is the $m \times m$ identity matrix and the $(N+1) \times(N+1)$ matrix $P$ is symmetric and positive definite. The same holds for $\bar{P}$, which thus is a valid norm $\|\mathbf{e}\|_{\bar{P}}^{2}=\mathbf{e}^{T} \bar{P} \mathbf{e}$. This leads to

$$
\left(\|\mathbf{e}\|_{\bar{P}}^{2}\right)_{t}=-\mathbf{e}^{T}\left(\bar{P} \bar{A}+(\bar{P} \bar{A})^{T}\right) \mathbf{e}
$$

Recall that $\bar{A}$ includes both the interior approximation and the boundary procedure. For (15) to be energy stable, the right-hand side (RHS) must be bounded by const. $\left(\|\mathbf{e}\|_{\bar{P}}^{2}\right)$, see [17] for more details on stability.

For a steady state to exist, we know from the previous section that the eigenvalues of $\bar{A}$ must have strictly positive real parts. We also know that if $\min _{i} \Re\left(\lambda_{i}\right)$ is large, the convergence will be fast. To manipulate the eigenvalues of $\bar{A}$ directly is almost impossible. However, by using the energy method, see (15), we can modify the matrix $(\bar{P} \bar{A})^{S}=\left(\bar{P} \bar{A}+(\bar{P} \bar{A})^{T}\right) / 2$ in such a way that it modifies $\bar{A}$ and most likely enhances the convergence rate.

In most cases, the internal part of the discretisation $(A)$ is given by the class of method chosen. The choice of boundary approximation $(\Sigma)$, on the other hand is crucial and will be considered in detail. As was mentioned above $(\Sigma=\Sigma(L))$ and it is therefore highly dependent on the boundary conditions in (4) but also on the specific numerical implementation technique. In this paper we focus on the latter.

Let us assume that we have chosen a particular boundary approximation such that $(\bar{P} \bar{A})^{S}$ is positive definite and leads to an energy decay in (15). Let $\lambda$ and $x$ be an eigenvalue and eigenvector to $\bar{A}$. By multiplying the relation $\bar{A} x=\lambda x$ from the left with $x^{*} \bar{P}$ and rearranging we get

$$
\lambda=\Re(\lambda)+i \Im(\lambda), \quad \Re(\lambda)=\frac{x^{*}(\bar{P} \bar{A})^{S} x}{x^{*} \bar{P} x}, \quad \Im(\lambda)=\frac{x^{*}(\bar{P} \bar{A})^{A S} x}{x^{*} \bar{P} x},
$$

where $(\bar{P} \bar{A})^{A S}=\left(\bar{P} \bar{A}-(\bar{P} \bar{A})^{T}\right) / 2$. Consequently, we can guarantee convergence to steady-state if we have an energy decay in $(15)$. We can also see from 
(16) that by making $(\bar{P} \bar{A})^{S}=\left(\bar{P} \bar{A}+(\bar{P} \bar{A})^{T}\right) / 2$ more positive definite, there is a possibility that we can increase the convergence rate $\min _{i} \Re\left(\lambda_{i}\right)$. For later reference we denote $\min _{i} \Re\left(\lambda_{i}\right)=$ the discrete decay rate.

The strategy in this paper for studying the influence of weak and strong solid wall boundary procedures can be summarized as follows. First we make sure that the continuous solid wall boundary conditions are well posed and lead to convergence to steady-state (otherwise further investigation is meaningless). Next we discretise and derive the different forms of $\bar{A}=A-\Sigma$. The inner scheme $A$ is the same for all cases but $\Sigma$ varies depending on the form of boundary implementation we use. We will study three types, the weak, the strong and an intermediate form of implementation. After that we use the energy method and try to determine if the schemes are stable. Finally we compute the eigenvalues of $\bar{A}$ and see which procedure yields the largest value of $\min _{i} \Re\left(\lambda_{i}\right)$.

\section{The Navier-Stokes equations}

The symmetrized frozen coefficient time-dependent compressible Navier-Stokes equation in two dimensions is given by (see $[1],[38]$ )

$$
U_{t}+\left(A_{1} U\right)_{x}+\left(A_{2} U\right)_{y}=\varepsilon\left[\left(B_{11} U_{x}+B_{12} U_{y}\right)_{x}+\left(B_{21} U_{x}+B_{22} U_{y}\right)_{y}\right] .
$$

In $(17), \varepsilon=1 / R e, U=\left[\bar{c} \rho^{\prime} /(\sqrt{\gamma} \bar{\rho}), u^{\prime}, v^{\prime}, T^{\prime} /\left(\bar{c} M_{\infty}^{2} \sqrt{\gamma(\gamma-1)}\right)\right]^{T}$ and

$$
\begin{aligned}
& A_{1}=\left[\begin{array}{cccc}
\bar{u} & \frac{\bar{c}}{\sqrt{\gamma}} & 0 & 0 \\
\frac{\bar{c}}{\sqrt{\gamma}} & \bar{u} & 0 & \sqrt{\frac{\gamma-1}{\gamma}} \bar{c} \\
0 & 0 & \bar{u} & 0 \\
0 & \sqrt{\frac{\gamma-1}{\gamma}} \bar{c} & 0 & \bar{u}
\end{array}\right], \quad A_{2}=\left[\begin{array}{cccc}
\bar{v} & 0 & \frac{\bar{c}}{\sqrt{\gamma}} & 0 \\
0 & \bar{v} & 0 & 0 \\
\frac{\bar{c}}{\sqrt{\gamma}} & 0 & \bar{v} & \sqrt{\frac{\gamma-1}{\gamma}} \bar{c} \\
0 & 0 & \sqrt{\frac{\gamma-1}{\gamma}} \bar{c} & \bar{v}
\end{array}\right], \\
& B_{11}=\left[\begin{array}{cccc}
0 & 0 & 0 & 0 \\
0 & \frac{\lambda+2 \mu}{\bar{\rho}} & 0 & 0 \\
0 & 0 & \frac{\mu}{\bar{\rho}} & 0 \\
0 & 0 & 0 & \frac{\gamma \mu}{\operatorname{Pr} \bar{\rho}}
\end{array}\right], B_{12}=B_{21}=\left[\begin{array}{cccc}
0 & 0 & 0 & 0 \\
0 & 0 & \frac{\lambda+\mu}{2 \bar{\rho}} & 0 \\
0 & \frac{\lambda+\mu}{2 \bar{\rho}} & 0 & 0 \\
0 & 0 & 0 & 0
\end{array}\right], B_{22}=\left[\begin{array}{cccc}
0 & 0 & 0 & 0 \\
0 & \frac{\mu}{\bar{\rho}} & 0 & 0 \\
0 & 0 & \frac{\lambda+2 \mu}{\bar{\rho}} & 0 \\
0 & 0 & 0 & \frac{\gamma \mu}{\operatorname{Pr} \bar{\rho}}
\end{array}\right] .
\end{aligned}
$$

The dependent variables consist of the density $\rho^{\prime}$, the velocities $u^{\prime}, v^{\prime}$ and the temperature $T^{\prime}$. The coefficients are frozen at a constant state $\bar{U}=[\bar{\rho}, \bar{u}, \bar{v}, \bar{T}]^{T}$. 
In the vectors and matrices above we have used the ratio of the specific heats $\gamma=c_{p} / c_{v}$, the speed of sound $\bar{c}$, the dynamic viscosity $\mu$, the bulk viscosity $\lambda$, the kinematic viscosity $\nu=\mu / \rho$, the Prandtl number $\operatorname{Pr}=\nu / \alpha$ ( $\alpha$ is the thermal diffusivity) and the Reynolds number $\operatorname{Re}=\rho_{\infty} U_{\infty} L / \mu_{\infty}$. The infinity subscript denotes free stream conditions and $L$ is a characteristic length. Note again that the form of the matrices (Jacobians) above are obtained for the symmetrized frozen coefficient version of the Navier-Stokes equations.

Equation (17) can be rewritten more compactly in the conservative form

$$
U_{t}+F_{x}+G_{y}=0
$$

where

$$
\begin{aligned}
& F=A_{1} U-\varepsilon\left(B_{11} U_{x}+B_{12} U_{y}\right)=F^{I}-\varepsilon F^{V} \\
& G=A_{2} U-\varepsilon\left(B_{21} U_{x}+B_{22} U_{y}\right)=G^{I}-\varepsilon G^{V}
\end{aligned}
$$

$F^{I}$ and $G^{I}$ contain the inviscid terms and $F^{V}$ and $G^{V}$ the viscous terms.

\subsection{The solid wall boundary conditions and well-posedness}

We consider the computational domain to be the half plane $\Omega \in(-\infty,+\infty) \times$ $[0,+\infty)$ and the solid wall to reside at $y=0$. Applying the energy method to (18) on the domain $\Omega$ and using the Green-Gauss theorem leads to

$$
\|U\|_{t}^{2}=\int_{-\infty}^{+\infty} U^{T}\left[G^{I}-2 \varepsilon G^{V}\right]_{y=0} d x-2 \varepsilon \iint_{\Omega}\left[\begin{array}{l}
U_{x} \\
U_{y}
\end{array}\right]^{T}\left[\begin{array}{ll}
B_{11} & B_{12} \\
B_{21} & B_{22}
\end{array}\right]\left[\begin{array}{l}
U_{x} \\
U_{y}
\end{array}\right] d x d y
$$

The contribution from the integral term is negative semi-definite since the

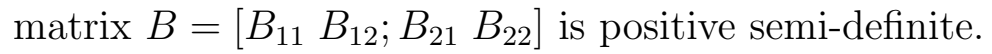

To limit the energy growth, the boundary term must be bounded using the correct number and form of boundary conditions. At a solid wall we are allowed three boundary conditions for the two-dimensional Navier-Stokes equations. By inserting zero velocity, i.e. $u^{\prime}=v^{\prime}=0$ we obtain $U^{T} G^{I}=\bar{v}\left(\tilde{\rho}^{2}+\tilde{T}^{2}\right)$ and $U^{T} G^{v}=\frac{\gamma \mu}{\operatorname{Pr} \bar{\rho}} T^{\prime} T_{y}^{\prime}$ where $\tilde{\rho}=\bar{c} \rho^{\prime} /(\sqrt{\gamma} \bar{\rho})$ and $\tilde{T}=T^{\prime} /\left(\bar{c} M_{\infty}^{2} \sqrt{\gamma(\gamma-1)}\right)$. Consequently, the boundary term is negative with $\bar{v} \leq 0$ and either $T^{\prime}=0$, $T_{y}^{\prime}=0$ or $T^{\prime}+\beta T_{y}^{\prime}=0, \beta>0$. We summarize the result in the following proposition.

Proposition 6.1 The continuous problem (17)-(19) with $\bar{v} \leq 0$, is well posed on the domain $(x, y) \in(-\infty,+\infty) \times[0,+\infty)$ if

$$
u^{\prime}=v^{\prime}=0 \quad \text { and } \quad T^{\prime}=0 \quad \text { or } \quad T_{y}^{\prime}=0, \quad \text { or } \quad T^{\prime}+\beta T_{y}^{\prime}=0, \quad \beta>0
$$




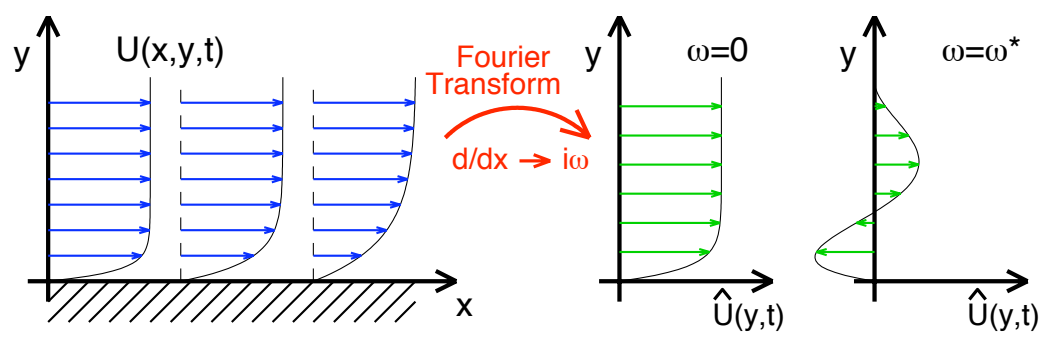

Fig. 3. A one-dimensional problem is obtained by using Fourier transform.

are used as solid wall boundary conditions at $y=0$.

Remark: The $n$-dimensional Navier-Stokes equations require $n$ boundary conditions at an inflow boundary and $n-1$ at an outflow boundary. In this case (two dimensions) we need four boundary conditions at an inflow boundary and three at an outflow boundary. The solid wall is in that sense a subsonic outflow boundary. For more discussions on boundary conditions see for example $[43],[17],[38]$

\section{Analysis of solid wall boundary procedures}

In this section we formulate a simplified but relevant model problem for the analysis of solid wall boundary procedures. The general theory outlined in section 2-5 will be applied.

\subsection{Formulation of the IBVP}

Consider the two dimensional equations given in (17). We are primarily interested in the phenomena occurring normal to the boundary at $y=0$ and Fourier transform in the $x$ direction to obtain

$$
\hat{U}_{t}+\mathcal{A} \hat{U}=0, \quad \mathcal{A}=\left(C_{0}+C_{1} \frac{\partial}{\partial y}+C_{2} \frac{\partial^{2}}{\partial^{2} y}\right) .
$$

Here $C_{0}=i \omega A_{1}-\varepsilon(i \omega)^{2} B_{11}, C_{1}=A_{2}-\varepsilon i \omega\left(B_{12}+B_{21}\right), C_{2}=-\varepsilon B_{22}$ and $\hat{U}(\omega, y, t)=\int_{-\infty}^{\infty} U(x, y, t) \exp (-i \omega x) d x$. That is, all $\partial / \partial x$ are replaced by $i \omega$, see Figure 3. Equation (21) is a one dimensional partial differential equation in the domain $0 \leq y \leq H$, where $H$ is considered large. We are interested in how the boundary treatment at $y=0$ affects the convergence to steady state. The parameter $\omega$ measures the size of the $x$ derivative in the original problem (17). To achieve an energy estimate we multiply (21) by $\hat{U}^{*}$, add the 
conjugate transpose, and integrate with respect to $y$. Note that the matrices $A_{1}, A_{2}, B_{11}, B_{12}, B_{21}$ and $B_{22}$ are symmetric and real-valued. We obtain

$$
\|\hat{U}\|_{t}^{2}+2 \varepsilon\|\hat{U}\|_{B}^{2}=\underbrace{\left[-\hat{U}^{*} A_{2} \hat{U}+\varepsilon\left(\hat{U}^{*} B_{22} \hat{U}_{y}+\hat{U}_{y}^{*} B_{22} \hat{U}\right)\right]_{0}^{H}}_{B T}
$$

where $\|\hat{U}\|^{2} \equiv \int_{0}^{H} \hat{U}^{*} \hat{U} d y$ and

$$
\|\hat{U}\|_{B}^{2}=\int_{0}^{H}\left[\begin{array}{c}
i \omega \hat{U} \\
\hat{U}_{y}
\end{array}\right]^{*} \underbrace{\left[\begin{array}{cc}
B_{11} & \left(B_{12}+B_{21}\right) / 2 \\
\left(B_{12}+B_{21}\right) / 2 & B_{22}
\end{array}\right]}_{B_{T O T}}\left[\begin{array}{c}
i \omega \hat{U} \\
\hat{U}_{y}
\end{array}\right] d y .
$$

The matrix $B_{T O T}$ is positive semi-definite. To bound the boundary terms $(B T)$ in (22) at the wall we use the no-slip boundary conditions $\hat{u}=\hat{v}=\hat{T}_{y}=0$.

Remark: In the following we focus on the results at $y=0$. At $y=H$, we use Dirichlet boundary conditions and a standard weak numerical implementation. That treatment can be found in Appendix A.

The solid wall boundary condition expressed on matrix form is

$$
L_{0} \hat{U}(0, t)+K_{0} \hat{U}(0, t)_{y}=0, \quad L_{0}=\left[\begin{array}{llll}
0 & 1 & 0 & 0 \\
0 & 0 & 1 & 0 \\
0 & 0 & 0 & 0
\end{array}\right], \quad K_{0}=\left[\begin{array}{llll}
0 & 0 & 0 & 0 \\
0 & 0 & 0 & 0 \\
0 & 0 & 0 & 1
\end{array}\right] .
$$

The number of rows in $L_{0}$ and $K_{0}$ corresponds to the number of boundary conditions given, and the number of columns is the same as the number of variables. The IBVP we will investigate in terms of convergence to steadystate is defined by (21) and (23) augmented with an initial condition. The formulation of this problem corresponds to (4). Proposition 6.1 guarantees that (21) in combination with (23) leads to a well-posed problem.

\subsection{The continuous decay rate}

To find the convergence rate we follow the procedure in Section 3.1, and Laplace transform (21) and (23). We obtain a version of (5) where $f=$ $\hat{U}(\omega, y, 0), L=L_{0}+K_{0} \partial / \partial y$ and $y$ is the independent variable. The ansatz $\hat{e}_{h}=\psi \exp (\kappa y)$ for the homogenous solution to (5) yields (7), where

$$
\mathcal{A}(\kappa)=i \omega A_{1}+\kappa A_{2}-\varepsilon\left((i \omega)^{2} B_{11}+i \omega \kappa\left(B_{12}+B_{21}\right)+\kappa^{2} B_{22}\right) .
$$


For non-trivial solutions we need to satisfy the second condition in (7), which for this particular problem means finding the roots of a $7^{\text {th }}$ degree polynomial in $\kappa$. The $\kappa_{j}$ and the corresponding $\psi_{j}$ are found numerically. Thereafter the homogenous solution is expressed as in (8), but scaled for $\Re(\kappa)>0$ for better conditioning. We have

$$
\hat{e}_{h}(\omega, y, s)=\sum_{\Re(\kappa)<0} \sigma_{j} \psi_{j} \exp \left(\kappa_{j} y\right)+\sum_{\Re(\kappa)>0} \sigma_{j} \psi_{j} \exp \left(\kappa_{j}(y-1)\right)=\Psi \bar{X}(y) \sigma
$$

where $\Psi, \bar{X}$ and $\sigma$ are given below. The conditions from both boundaries are used to obtain (9). See Appendix A for the upper boundary operators $L_{N}$ and $K_{N}$. Here $E(s) \sigma=\hat{g}$ is a $7 \times 7$ system of equations, where

$$
\begin{aligned}
& E(s)=\left[\begin{array}{c}
L_{0} \Psi \bar{X}(0)+K_{0} \Psi \bar{K} \bar{X}(0) \\
L_{N} \Psi \bar{X}(1)+K_{N} \Psi \bar{K} \bar{X}(1)
\end{array}\right], \\
& \bar{X}(y)=\operatorname{diag}\left[e^{\kappa_{1} y}, e^{\kappa_{2} y}, e^{\kappa_{3} y}, e^{\kappa_{4}(y-1)}, e^{\kappa_{5}(y-1)}, e^{\kappa_{6}(y-1)}, e^{\kappa_{7}(y-1)}\right], \\
& \Psi=\left[\psi_{1}, \ldots, \psi_{7}\right], \quad \bar{K}=\operatorname{diag}\left[\kappa_{1}, \ldots, \kappa_{7}\right], \quad \sigma=\left[\sigma_{1}, \ldots, \sigma_{7}\right]^{T} .
\end{aligned}
$$

To find the decay rate we solve for the $s$ values such that $|E(s)|=0$, using the secant method. This is done repeatedly for a broad band of starting points and these $s$ values form the continuous spectrum of the problem. The real part $\eta^{*}$ of the $s$ furthest to the right, such that $\eta^{*} \geq \Re(s) \forall s$, is the continuous decay rate, which gives the convergence rate to steady-state, see equation (10).

\subsection{Formulation of the IVP}

We introduce an equidistant mesh $y_{j}=H j / N$, where $j=0,1, \ldots, N$ and $N+1$ is the number of grid points. Let $V$ be the semi-discrete representation of $\hat{U}$ in $(21)$ such that $V_{j}(t) \approx \hat{U}\left(y_{j}, t\right)$. The semi-discrete representation of the $\operatorname{IBVP}(21),(23)$ is

$$
V_{t}+A V=\Sigma_{0} V, \quad A=\left[\left(I_{N} \otimes C_{0}\right)+\left(D_{1} \otimes C_{1}\right)+\left(D_{2} \otimes C_{2}\right)\right],
$$

where $I_{N}$ is the $(N+1) \times(N+1)$ identity matrix. The operator $D_{1} \equiv P^{-1} Q$ mimics $\partial / \partial y$ and $D_{2} \equiv P^{-1} M$ mimics $\partial^{2} / \partial y^{2}$. The term $\Sigma_{0} V$ takes care of the boundary procedures at the solid wall. (The treatment of the upper boundary is neglected here but described in Appendix A.) The IVP we will investigate in terms of convergence to steady-state is (24). The relation (24) augmented with an initial condition corresponds to (12).

The operators $D_{1}, D_{2}$ presented above are on Summation-By-Parts (SBP) 
form and have the following properties

$$
\begin{array}{ll}
Q+Q^{T}=E_{N}-E_{0} & P=P^{T}>0 \\
M=-S^{T} R S+\left(E_{N}-E_{0}\right) S & R+R^{T} \geq 0
\end{array}
$$

where $E_{0}=\operatorname{diag}(1,0, \ldots, 0)$ and $E_{N}=\operatorname{diag}(0, \ldots, 0,1)$. For more details on SBP operators, see $[42,27]$. If $D_{2}=D_{1}^{2}$ is used, then $S=D_{1}, R=P$.

\subsection{The discrete energy method}

Next we multiply (24) by $V^{*} \bar{P}$ from the left, where $\bar{P}=P \otimes I_{4}$, and add the conjugate transpose. Note that since $\bar{P}$ is positive definite it gives rise to a valid norm $\|V\|_{\bar{P}}^{2}=V^{*} \bar{P} V$, corresponding to the continuous norm $\|\hat{U}\|^{2}=$ $\int_{0}^{H} \hat{U}^{*} \hat{U} d y$. Using the properties in (25) we obtain

$$
\begin{gathered}
\frac{d}{d t}\|V\|_{\bar{P}}^{2}+2 \varepsilon\left[\begin{array}{c}
i \omega V \\
\bar{D} V
\end{array}\right]^{*} \underbrace{\left[\begin{array}{cc}
\left(P \otimes B_{11}\right) & P \otimes\left(B_{12}+B_{21}\right) / 2 \\
P \otimes\left(B_{12}+B_{21}\right) / 2 & \left(P \otimes B_{22}\right)
\end{array}\right]}_{B_{T O T}^{\text {disc. }}}\left[\begin{array}{c}
i \omega V \\
\bar{D} V
\end{array}\right] \\
\leq V_{0}^{*} A_{2} V_{0}-\varepsilon\left(V_{0}^{*} B_{22}(\bar{D} V)_{0}+(\bar{D} V)_{0}^{*} B_{22} V_{0}\right)+V^{*}\left(\bar{P} \Sigma_{0}+\Sigma_{0}^{*} \bar{P}\right) V .
\end{gathered}
$$

In (26) we have used $D_{2}=D_{1}^{2}$ and $\bar{D} \equiv D_{1} \otimes I_{4}$. We have also assumed a dissipative treatment of the upper boundary and indicated that by the inequality sign. The estimate in (26) is similar to the continuous estimate in (22), except for the terms including $\Sigma_{0}$. The estimate (26) also corresponds to the general formulation (15).

$P$ and $B_{11}$ are square matrices and hence $P \otimes B_{11}$ and $B_{11} \otimes P$ are permutation similar, meaning that there exists a permutation matrix $\Phi$ such that $P \otimes B_{11}=$ $\Phi^{T}\left(B_{11} \otimes P\right) \Phi$, see [35], [21]. The same holds for $B_{12}, B_{21}$ and $B_{22}$. As a consequence we can write $B_{T O T}^{\text {disc. }}=\left(I_{2} \otimes \Phi\right)^{T}\left(B_{T O T} \otimes P\right)\left(I_{2} \otimes \Phi\right)$. Further, we can split $P$ into $P=\widetilde{P}+p_{0} E_{0}$ such that (26) becomes

$$
\frac{d}{d t}\|V\|_{\bar{P}}^{2}+2 \varepsilon\left[\begin{array}{l}
i \omega \Phi V \\
\Phi \bar{D} V
\end{array}\right]^{*}\left(B_{T O T} \otimes \widetilde{P}\right)\left[\begin{array}{l}
i \omega \Phi V \\
\Phi \bar{D} V
\end{array}\right] \leq B T_{0}
$$


where

$$
\begin{aligned}
B T_{0} & =\left[\begin{array}{c}
V_{0} \\
i \omega V_{0} \\
(\bar{D} V)_{0}
\end{array}\right]^{*}\left[\begin{array}{ccc}
A_{2} & 0 & -\varepsilon B_{22} \\
0 & -2 p_{0} \varepsilon B_{11} & -p_{0} \varepsilon\left(B_{12}+B_{21}\right) \\
-\varepsilon B_{22} & -p_{0} \varepsilon\left(B_{12}+B_{21}\right) & -2 p_{0} \varepsilon B_{22}
\end{array}\right]\left[\begin{array}{c}
V_{0} \\
i \omega V_{0} \\
(\bar{D} V)_{0}
\end{array}\right] \\
& +V^{*}\left(\bar{P} \Sigma_{0}+\Sigma_{0}^{*} \bar{P}\right) V .
\end{aligned}
$$

If $\widetilde{P}, B_{T O T} \geq 0$ then $B_{T O T} \otimes \widetilde{P} \geq 0$, which makes the quadratic term in (27) dissipative. Thus we need $\widetilde{P} \geq 0$ for stability and for a second order accurate scheme this implies that $p_{0} \leq \Delta y / 2$.

The interior discretization is the same for all three schemes, and in the numerical simulations we use the compact second order stencil. The boundary treatment is included in the terms involving $\Sigma_{0}$ in (28). $\Sigma_{0}$ will be different dependent on whether the boundary procedure is weak, strong or a mixture of both. If $B T_{0} \leq 0$ the numerical scheme is guaranteed to be stable.

\subsubsection{A weak solid wall boundary procedure}

Consider the penalty term

$$
\Sigma_{0}^{\text {weak }} V=\left(P^{-1} e_{0} \otimes \tau_{0}\right)\left(L_{0} V_{0}+K_{0}(\bar{D} V)_{0}\right)
$$

where the boundary operators are given in $(23)$ and where $e_{0}=[1,0, \ldots, 0]^{T}$. The dimension of $\tau_{0}$ is the transpose of the dimensions of $L_{0}$ and $K_{0}$, which means that $\tau_{0}$ is a $4 \times 3$ matrix. We insert the term $V^{*}\left(\bar{P} \Sigma_{0}^{\text {weak }}+\left(\Sigma_{0}^{\text {weak }}\right)^{*} \bar{P}\right) V$ into the matrix in (28) and obtain

$$
B T_{0}^{\text {weak }}=[\begin{array}{c}
V_{0} \\
i \omega V_{0} \\
(\bar{D} V)_{0}
\end{array} \underbrace{\left[\begin{array}{ccc}
\tau_{0} L_{0}+\left(\tau_{0} L_{0}\right)^{*}+A_{2} & 0 & \tau_{0} K_{0}-\varepsilon B_{22} \\
0 & -2 p_{0} \varepsilon B_{11} & -p_{0} \varepsilon\left(B_{12}+B_{21}\right) \\
\left(\tau_{0} K_{0}\right)^{*}-\varepsilon B_{22} & -p_{0} \varepsilon\left(B_{12}+B_{21}\right) & -2 p_{0} \varepsilon B_{22}
\end{array}\right]}_{M_{0}^{\text {weak }}}\left[\begin{array}{c}
V_{0} \\
i \omega V_{0} \\
(\bar{D} V)_{0}
\end{array}\right] .
$$

To prevent energy growth we need the $12 \times 12$ matrix $M_{0}^{\text {weak }}$ to be negative semi-definite. By choosing $\tau_{14}=\tau_{24}=\tau_{34}=\tau_{12}=\tau_{23}=\tau_{32}=\tau_{42}=0$ and $\tau_{13}=-\bar{c} / \sqrt{\gamma}, \tau_{43}=-\bar{c} \sqrt{(\gamma-1) / \gamma}$ in the penalty matrix $\tau_{0}$, the matrix $M_{0}^{\text {weak }}$ 
decouples into three parts as

$$
\begin{aligned}
& B T_{0}^{\text {weak }}=\left[\begin{array}{c}
\rho_{0} \\
T_{0} \\
i \omega T_{0} \\
(D T)_{0}
\end{array}\right]^{*} \underbrace{\left[\begin{array}{cccc}
\theta_{11} & 0 & 0 & 0 \\
0 & \theta_{22} & 0 & \theta_{24} \\
0 & 0 & \theta_{33} & 0 \\
0 & \theta_{42} & 0 & \theta_{44}
\end{array}\right]}_{M_{0}^{\theta}}\left[\begin{array}{c}
\rho_{0} \\
T_{0} \\
i \omega T_{0} \\
(D T)_{0}
\end{array}\right] \\
& +\left[\begin{array}{c}
u_{0} \\
i \omega v_{0} \\
(D u)_{0}
\end{array}\right]^{*} \underbrace{\left[\begin{array}{ccc}
\psi_{11} & 0 & \psi_{13} \\
0 & \psi_{22} & \psi_{23} \\
\psi_{31} & \psi_{32} & \psi_{33}
\end{array}\right]}_{M_{0}^{\psi}}\left[\begin{array}{c}
u_{0} \\
i \omega v_{0} \\
(D u)_{0}
\end{array}\right]+\left[\begin{array}{c}
v_{0} \\
i \omega u_{0} \\
(D v)_{0}
\end{array}\right]^{\left[\begin{array}{ccc}
\varphi_{11} & 0 & \varphi_{13} \\
0 & \varphi_{22} & \varphi_{23} \\
\varphi_{31} & \varphi_{32} & \varphi_{33}
\end{array}\right]}\left[\begin{array}{c}
v_{0} \\
i \omega u_{0} \\
(D v)_{0}
\end{array}\right]
\end{aligned}
$$

where we have used the notation $V_{i}=\left[\begin{array}{lll}\rho_{i} & u_{i} & v_{i}\end{array} T_{i}\right]^{T}$ and where

$$
\begin{aligned}
& \psi_{11}=\bar{v}+\tau_{22}+\tau_{22}^{*} \quad \varphi_{11}=\bar{v}+\tau_{33}+\tau_{33}^{*} \quad \theta_{11}=\bar{v} \\
& \psi_{13}=-\varepsilon \frac{\mu}{\bar{\rho}} \quad \varphi_{13}=-\varepsilon \frac{\lambda+2 \mu}{\bar{\rho}} \quad \theta_{22}=\bar{v} \\
& \psi_{22}=-2 p_{0} \varepsilon \frac{\mu}{\bar{\rho}} \quad \varphi_{22}=-2 p_{0} \varepsilon \frac{\lambda+2 \mu}{\bar{\rho}} \quad \theta_{33}=-2 p_{0} \varepsilon \frac{\gamma \mu}{\operatorname{Pr} \bar{\rho}} \\
& \psi_{23}=-p_{0} \varepsilon \frac{\lambda+\mu}{\bar{\rho}} \quad \varphi_{23}=-p_{0} \varepsilon \frac{\lambda+\mu}{\bar{\rho}} \quad \theta_{24}=\tau_{44}-\varepsilon \frac{\gamma \mu}{\operatorname{Pr} \bar{\rho}} \\
& \psi_{33}=-2 p_{0} \varepsilon \frac{\mu}{\bar{\rho}} \quad \varphi_{33}=-2 p_{0} \varepsilon \frac{\lambda+2 \mu}{\bar{\rho}} \quad \theta_{44}=-2 p_{0} \varepsilon \frac{\gamma \mu}{\operatorname{Pr} \bar{\rho}} .
\end{aligned}
$$

The choice $\tau_{44}=\varepsilon \gamma \mu /\left(P_{r} \bar{\rho}\right)$ makes the sub-matrix $M_{0}^{\theta}$ diagonal with negative entries. To make $M_{0}^{\psi} \leq 0$ we define $\widetilde{M}_{0}^{\psi} \equiv \Xi M_{0}^{\psi} \Xi^{T}$, such that $\widetilde{M}_{0}^{\psi}$ is block diagonal. Next $\tau_{22}$ is chosen such that $\widetilde{M}_{0}^{\psi}$, and consequently $M_{0}^{\psi}$, is negative semi-definite. The same procedure is repeated for the sub-matrix $M_{0}^{\varphi}$. We have now proved the result presented in the following proposition.

Proposition 7.1 The IVP (24) with the penalty term $\Sigma_{0}$ defined by (29) yields a stable scheme if

$$
\tau_{0}=\left[\begin{array}{ccc}
0 & -\bar{c} / \sqrt{\gamma} & 0 \\
\tau_{22} & 0 & 0 \\
0 & \tau_{33} & 0 \\
0 & -\bar{c} \sqrt{\frac{\gamma-1}{\gamma}} \tau_{44}
\end{array}\right] \quad \begin{aligned}
& \tau_{22} \leq-\bar{v} / 2-\varepsilon \mu^{3} /\left(p_{0} \bar{\rho}(\lambda+3 \mu)(\mu-\lambda)\right) \\
& \tau_{33} \leq-\bar{v} / 2-\varepsilon(\lambda+2 \mu)^{3} /\left(p_{0} \bar{\rho}(\lambda+3 \mu)(3 \lambda+5 \mu)\right) \\
& \tau_{44}=\varepsilon \gamma \mu /\left(P_{r} \bar{\rho}\right)
\end{aligned}
$$

where $p_{0} \leq \Delta y / 2$. 
Note that the penalty scales with the number of grid points. We will use the limit values for $\tau_{22}, \tau_{33}$ and $p_{0}=\Delta y / 2$ throughout our numerical simulations.

\subsubsection{A mixed solid wall boundary procedure}

Here the boundary conditions $\hat{u}(0, t)=\hat{v}(0, t)=0$ are imposed strongly, while $\hat{T}_{y}(0, t)=0$ is imposed weakly. The latter is managed by the penalty term

$$
\Sigma_{0}^{T_{y}} V=\left(P^{-1} e_{0} \otimes \tilde{\tau}_{0}\right) \widetilde{K}_{0}(\bar{D} V)_{0},
$$

where $\tilde{\tau}_{0}=\left[0,0,0, \tau_{44}\right]^{T}$ is equal to the third column of $\tau_{0}$ in Proposition 7.1 and $\widetilde{K}_{0}=[0,0,0,1]$ is the third row of $K_{0}$ in $(23)$.

In the strong boundary procedure we use $u_{0}=v_{0} \equiv 0$, and there is no need for an update. The corresponding rows and columns are cancelled from the system of equations. The resulting system for the mixed scheme is two rows/columns smaller than the system obtained using the weak boundary procedure.

For analysis purposes the scheme is expanded such that the left-hand side is equal to that of (24), and identical terms are added to the right-hand side of the scheme. The equation

$$
V_{t}+A V=\Sigma_{0}^{m i x} V, \quad \Sigma_{0}^{m i x} V=\Sigma_{0}^{u, v} V+\Sigma_{0}^{T_{y}} V
$$

is identical to the original mixed scheme (except for two empty "dummy" rows and columns). Here $\Sigma_{0}^{u, v}$ consists of the terms added to cancel the influence from $u_{0}, v_{0}$ in row/column two and three of $A$. The upper 8 x8 corner of $\Sigma_{0}^{u, v}$ is given below, the rest of the matrix is zero.

$$
\Sigma_{0}^{u, v}=\left[\begin{array}{cccc|cccc}
0 & i \omega a & \frac{-a}{\Delta y} & 0 & 0 & 0 & 0 & 0 \\
i \omega a & i \omega \bar{u}+\varepsilon \omega^{2} \varphi-\frac{\bar{v}}{\Delta y} & \frac{\varepsilon i \omega(\varphi-\psi)}{\Delta y} & i \omega b & 0 & \frac{\bar{v}}{\Delta y} & \frac{-\varepsilon i \omega(\varphi-\psi)}{\Delta y} & 0 \\
\frac{-a}{\Delta y} & \frac{\varepsilon i \omega(\varphi-\psi)}{\Delta y} & i \omega \bar{u}+\varepsilon \omega^{2} \psi-\frac{\bar{v}}{\Delta y} & \frac{-b}{\Delta y} & \frac{a}{\Delta y} \frac{-\varepsilon i \omega(\varphi-\psi)}{\Delta y} & \frac{\bar{v}}{\Delta y} & \frac{b}{\Delta y} \\
0 & i \omega b & \frac{-b}{\Delta y} & 0 & 0 & 0 & 0 & 0 \\
\hline 0 & 0 & \frac{-a}{2 \Delta y} & 0 & & & \\
0 & \frac{-\bar{v}}{2 \Delta y}-\frac{\varepsilon \psi}{\Delta y^{2}} & \frac{\varepsilon i \omega(\varphi-\psi)}{2 \Delta y} & 0 & & & \\
0 & \frac{\varepsilon i \omega(\varphi-\psi)}{2 \Delta y} & \frac{-\bar{v}}{2 \Delta y}-\frac{\varepsilon \varphi}{\Delta y^{2}} & 0 & & & 0 & \\
0 & 0 & \frac{-b}{2 \Delta y} & 0 & & & \ddots
\end{array}\right]
$$

The abbreviations $a=\bar{c} / \sqrt{\gamma}, b=\bar{c} \sqrt{(\gamma-1) / \gamma}, \varphi=(\lambda+2 \mu) / \bar{\rho}$ and $\psi=\mu / \bar{\rho}$ have been used to shorten the expressions above.

The final boundary term $B T_{0}^{\text {mix }}$ corresponds to (28). Using the $\tilde{\tau}_{0}$ specified above, the boundary terms decouples into three independent parts (corre- 
spondingly to the weak case, see (30)). Thus, to make $B T_{0}^{\text {mix }} \leq 0$ the three sub-matrices

$$
M_{0}^{\theta}=\left[\begin{array}{cccc}
\theta_{11} & 0 & 0 & 0 \\
0 & \theta_{22} & 0 & 0 \\
0 & 0 & \theta_{33} & 0 \\
0 & 0 & 0 & \theta_{44}
\end{array}\right], M_{0}^{\psi}=\left[\begin{array}{ccc}
\psi_{11} & 0 & \psi_{13} \\
0 & \psi_{22} & \psi_{23} \\
\psi_{31} & \psi_{32} & \psi_{33}
\end{array}\right], M_{0}^{\varphi}=\left[\begin{array}{ccc}
\varphi_{11} & 0 & \varphi_{13} \\
0 & \varphi_{22} & \varphi_{23} \\
\varphi_{31} & \varphi_{32} & \varphi_{33}
\end{array}\right]
$$

must be negative semi-definite. $M_{0}^{\theta}$ is identical to the weak version and hence negative semi-definite. For the other two sub-matrices we have

$$
\begin{array}{ll}
\psi_{11}=-2 \varepsilon \frac{\mu}{\bar{\rho} \Delta y} & \varphi_{11}=-2 \varepsilon \frac{\lambda+2 \mu}{\bar{\rho} \Delta y} \\
\psi_{13}=-2 \varepsilon \frac{\mu}{\bar{\rho}} & \varphi_{13}=-2 \varepsilon \frac{\lambda+2 \mu}{\bar{\rho}} \\
\psi_{22}=\left(\Delta y-2 p_{0}\right) \varepsilon \frac{\mu}{\bar{\rho}} & \varphi_{22}=\left(\Delta y-2 p_{0}\right) \varepsilon \frac{\lambda+2 \mu}{\bar{\rho}} \\
\psi_{23}=\left(\Delta y-p_{0}\right) \varepsilon \frac{\lambda+\mu}{\bar{\rho}} & \varphi_{23}=\left(\Delta y-p_{0}\right) \varepsilon \frac{\lambda+\mu}{\bar{\rho}} \\
\psi_{33}=-2 p_{0} \varepsilon \frac{\mu}{\bar{\rho}} & \varphi_{33}=-2 p_{0} \varepsilon \frac{\lambda+2 \mu}{\bar{\rho}}
\end{array}
$$

Note that $M_{0}^{\psi}$ multiplies $\left[u_{0}, i \omega v_{0},\left(D_{1} u\right)_{0}\right]^{T}$ where both $u_{0}$ and $v_{0}$ are identically zero. Thus the only element of $M_{0}^{\psi}$ we need to consider is $\psi_{33}$ which is clearly negative. The same arguments hold for $M_{0}^{\varphi}$ and hence the mixed scheme is energy stable. We summarize the result in the following proposition.

Proposition 7.2 The IVP (24) with the penalty parameter $\Sigma_{0}=\Sigma_{0}^{u, v}+\Sigma_{0}^{T_{y}}$ from (32), where $\Sigma_{0}^{u, v}$ and $\Sigma_{0}^{T_{y}}$ are specified in (31) and (33), yields a stable scheme if

is used.

$$
\tilde{\tau}_{0}=\left[0,0,0, \varepsilon \gamma \mu /\left(P_{r} \bar{\rho}\right)\right]^{T}
$$

\subsubsection{A strong solid wall boundary procedure}

Here, all the conditions $\hat{u}(0, t)=0, \hat{v}(0, t)=0$ and $\hat{T}_{y}(0, t)=0$ are imposed strongly. The last condition is implemented as $T_{1}-T_{0}=0$. As for the mixed scheme $u_{0}$ and $v_{0}$ will not be updated and the corresponding rows and columns in the system of equations are cancelled. $T_{0}$ is also redundant since $T_{0}=T_{1}$. Hence the strong system has three equations less than the weak system. 
For analysis purposes we expanded the strong scheme to the same size as the weak and mixed scheme. The left-hand side became equal to that of (24) by adding $\Sigma_{0}^{\text {strong }} V$ to both sides of the original strong scheme. The expanded strong scheme was written

$$
V_{t}+A V=\Sigma_{0}^{\text {strong }} V
$$

where $\Sigma_{0}^{\text {strong }} V$ is given in Appendix B. However, despite a considerable effort, the strong scheme can not be proven energy stable in the $\bar{P}$ norm. This has consequences, as we will see exemplified later.

\section{Numerical simulations}

All three schemes (weak, mixed and strong) in the previous section, can be written on the form $V_{t}+\bar{A} V=0$. As already mentioned in section 5.1, a large value of the discrete decay rate $\min _{i}\left(\Re\left(\lambda_{i}\right)\right)$, where $\lambda_{i}$ are the eigenvalues of $\bar{A}$, will give fast convergence to steady state. We will first compute the eigenvalues of $\bar{A}$ for the linear IVP (24) and investigate which of the three solid wall boundary procedures that leads to the fastest convergence to steady-state. Next we will check if the conclusions drawn from the linear model problem carries over to the fully nonlinear Navier-Stokes equations.

\subsection{The discrete decay rate}

We have computed the discrete decay rate for various values of $\omega, \varepsilon$ and $\bar{v}$. In all cases we have used $\bar{u}=1$ as the mean velocity in the $x$ direction, $M=0.5$ as the Mach number, $\bar{\rho}=1$ as the mean density, $\bar{c}=1 / M$ as the speed of sound, $\gamma=1.4$, the dynamic viscosity $\mu=1$, the bulk viscosity $\lambda=-2 / 3$ and $P_{r}=0.7$ as the Prandtl number.

Firstly, the $x$ dependence (represented by $\omega$ ) was varied, while the parameters $\varepsilon$ and $\bar{v}$ were fixed. In Figure $4, \varepsilon=0.1$ and $\bar{v}=-0.1$ was used and the discrete decay rate for two typical meshes is shown. The discrete decay rates obtained by the weak procedure is larger than the ones for the two other formulations. Note in particular that small values of $\omega$ lead to small values of the discrete decay rate.

In Figure 4 one can see that the numerical decay rates converge to the continuous ones when the mesh is refined, for small $\omega$. To resolve also the higher modes, an even finer mesh would be required. In Figure 5, the same calculations are performed for $\varepsilon=0.01$ and $\bar{v}=-0.1$. The weak scheme still produces decay rates larger than the ones from the other two formulations. 


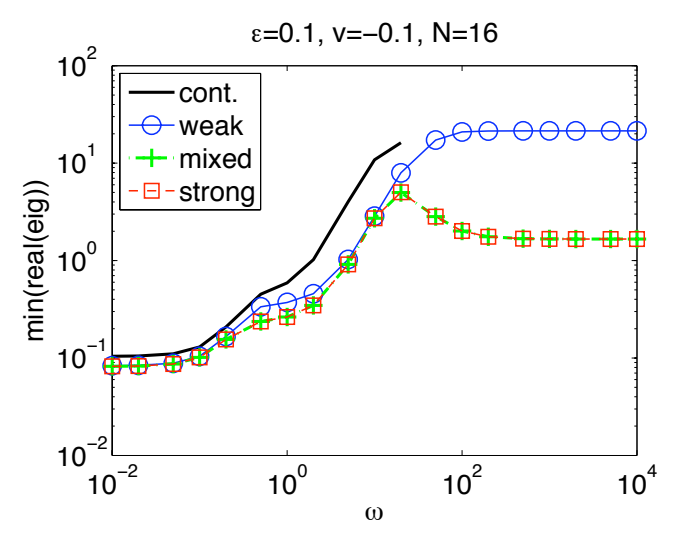

(a) $N=16$

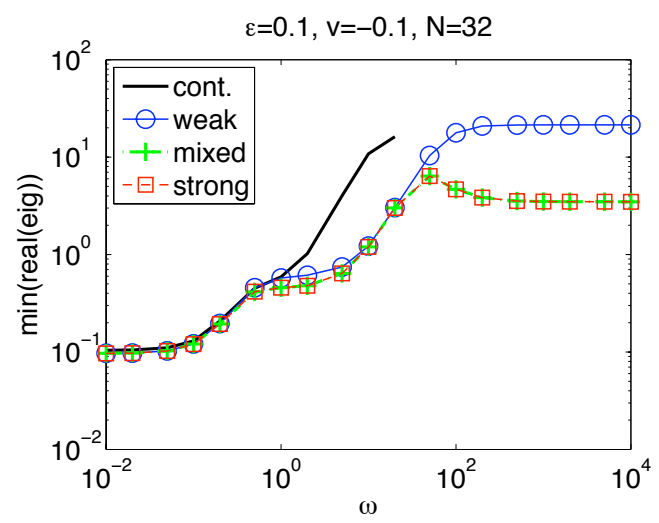

(b) $N=32$

Fig. 4. $\min _{i}\left(\Re\left(\lambda_{i}\right)\right)$ for $\varepsilon=0.1, \bar{v}=-0.1$. Varying $\omega$.

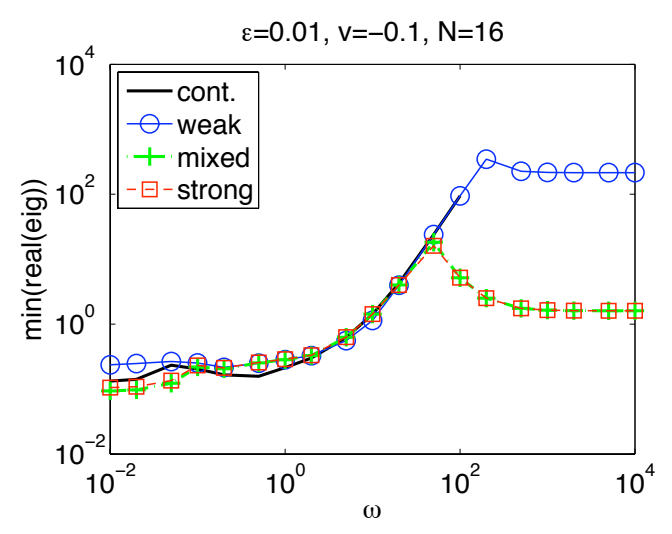

(a) $N=16$

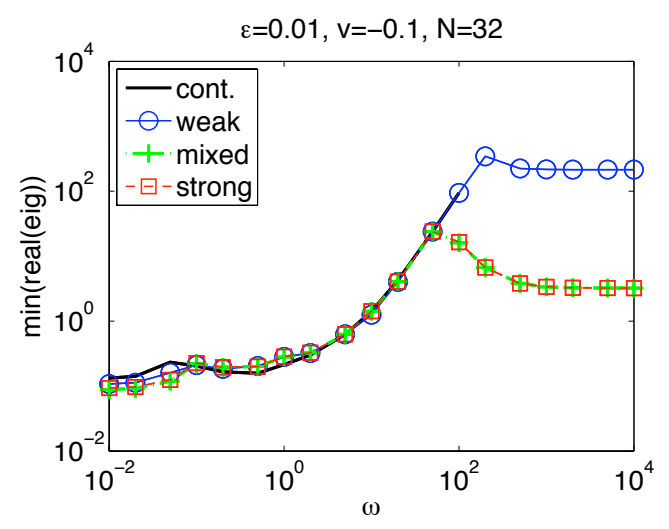

(b) $N=32$

Fig. 5. $\min _{i}\left(\Re\left(\lambda_{i}\right)\right)$ for $\varepsilon=0.01, \bar{v}=-0.1$. Varying $\omega$.

As before small values of $\omega$ gives the smallest (i.e. worst) values of the decay rate $\min _{i}\left(\Re\left(\lambda_{i}\right)\right)$.

The results in Figure 4 and 5 indicate that $\omega=0$ gives the smallest values of the decay rates, i.e. $\omega=0$ is the worst and most important case to study from a steady state computations point of view. In the following we focus on that case.

In Figure 6 we show $\min _{i}\left(\Re\left(\lambda_{i}\right)\right)$ for $\omega=0$ for varying values of $\varepsilon, \bar{v}$ and the number of grid points. The weak scheme has distinctly larger values of the decay rate than the other two schemes for coarse meshes. Recall that a large positive value of the discrete decay rate means fast convergence to steady state. For example, the results in Figure 6(b) indicate that the convergence to steady-state is five times faster than for the mixed and strong scheme. For fine meshes the discrete decay rate for all the schemes converge to the continuous decay rate, which in the figures is indicated by a solid black line. 


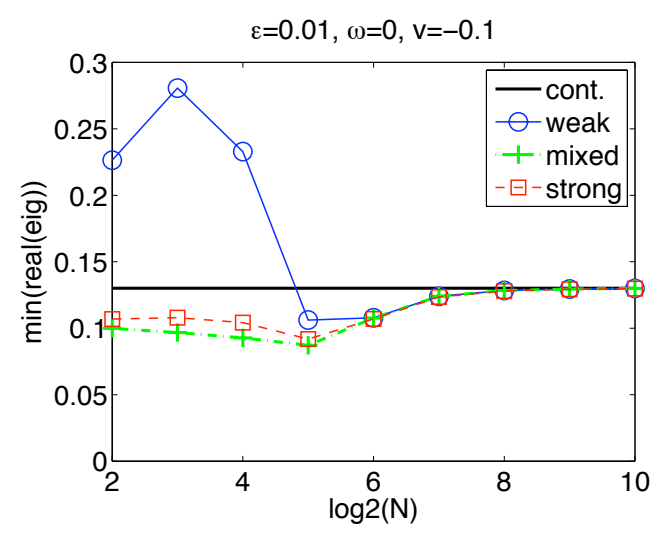

(a) $\varepsilon=0.01, \bar{v}=-0.1$

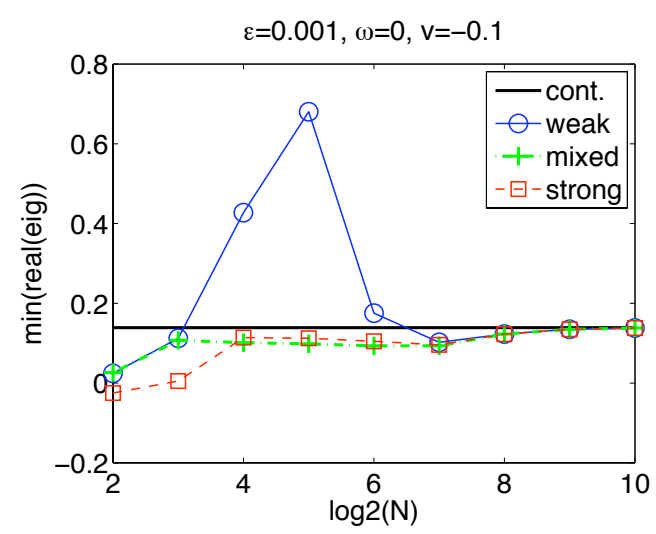

(c) $\varepsilon=0.001, \bar{v}=-0.1$

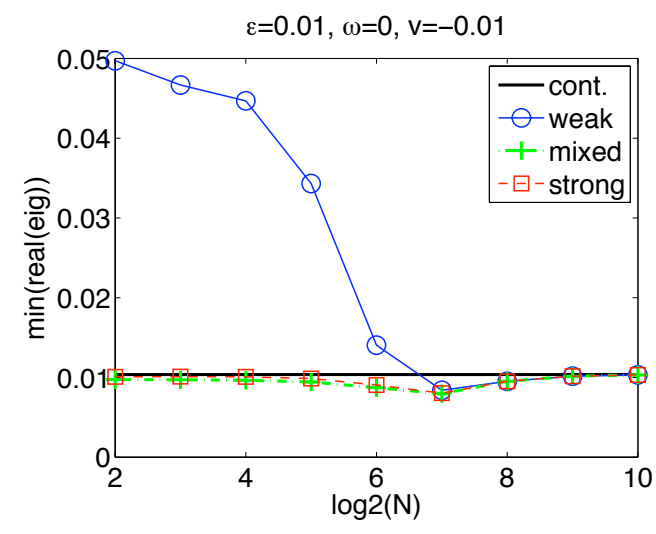

(b) $\varepsilon=0.01, \bar{v}=-0.01$

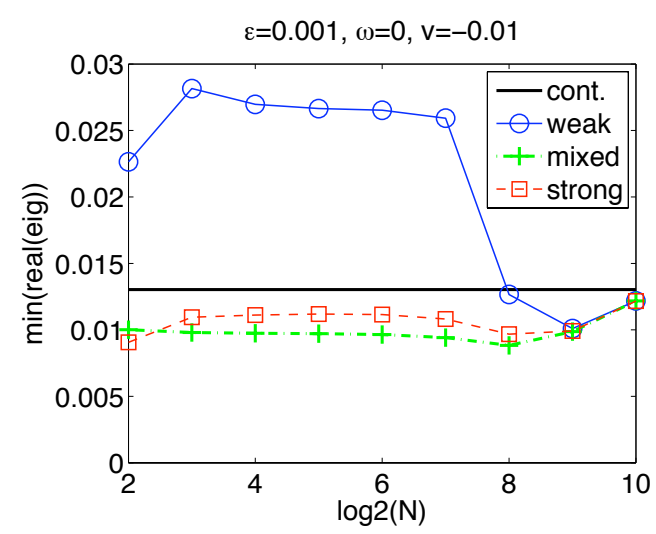

(d) $\varepsilon=0.001, \bar{v}=-0.01$

Fig. 6. $\min _{i}\left(\Re\left(\lambda_{i}\right)\right)$ for $\omega=0$.

Note that the strong scheme has $\min _{i}\left(\Re\left(\lambda_{i}\right)\right)<0$ at one point, in Figure 6(c). In that particular case the strong scheme will not yield a bounded solution for long time simulations. A stable numerical procedure for both the weak and the mixed scheme is guaranteed, see Propositions 7.1 and 7.2, but for the strong scheme no such guarantee exists.

\subsection{Numerical results using a fully nonlinear finite volume solver}

The analysis of our model problem indicates that the weak scheme yields faster convergence to steady-state. To investigate if this result carries over to the fully nonlinear Navier-Stokes equations, we use the finite volume solver Edge, which is applicable on both structured and unstructured grids, see [9], [11]. The governing equations are integrated explicitly with a multistage Runge-Kutta scheme to steady-state and acceleration by FAS agglomeration multigrid can be used. There are numerous boundary conditions in Edge for walls, external boundaries and periodic boundaries.

In Figure 7(a-c) the meshes used in this section for the first flow case are shown, 


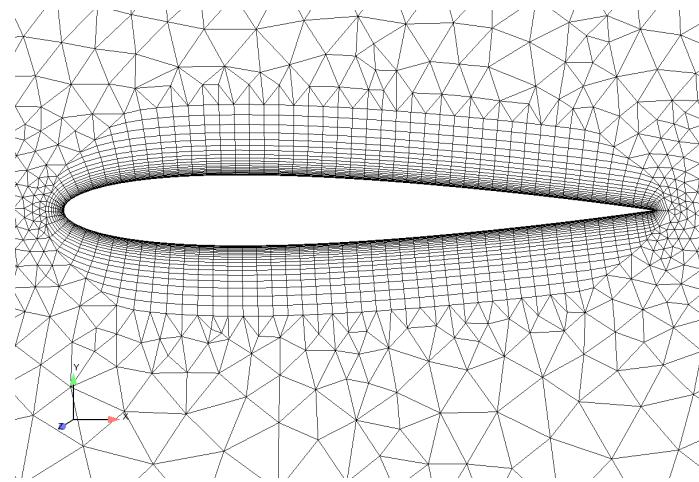

(a) Coarse mesh

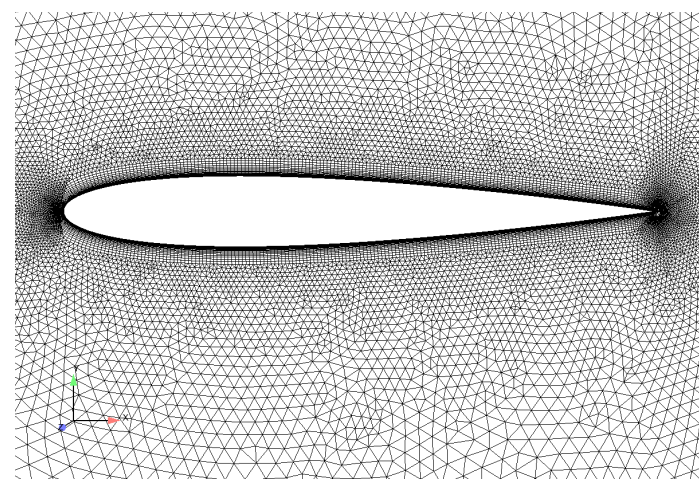

(c) Fine mesh

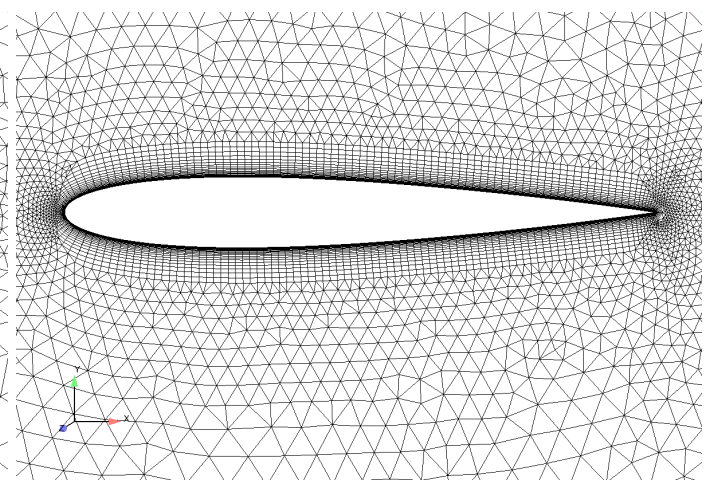

(b) Medium mesh

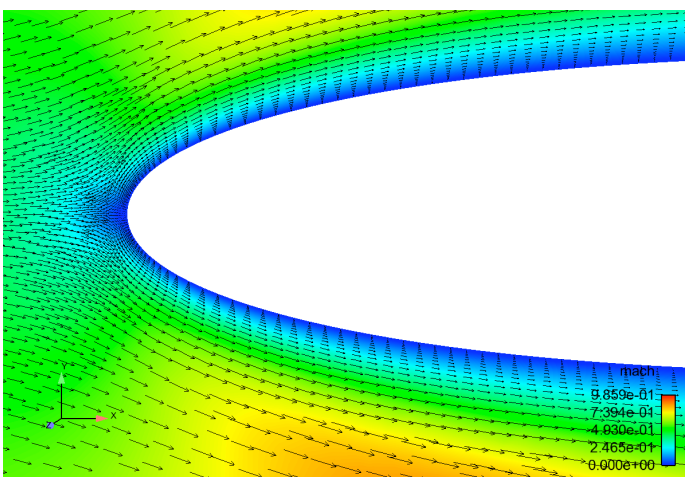

(d) Velocity field

Fig. 7. The NACA0012 geometry and a flow field.

and in Figure 7(d), the flow field around the nose of the NACA0012 airfoil is shown. The flow conditions are as follows: Mach number $M=0.8$, Reynolds number $R e=500$ and $\alpha=0^{\circ}$ as the angle of attack. These parameters yield a subsonic, low Reynolds flow which is assumed laminar.

Figure 8 displays the convergence to steady-state using the density residual. No artificial dissipation is used. The weak solution converges faster than the solutions obtained by the other two schemes. The difference is more pronounced for the coarsest grid, which is what is expected from the linear results. Next we investigate the flow over a flat plate with the geometry schematically depicted in Figure 9(a). For $x<0$ a symmetry condition is used, and for $x \geq 0$ the no-slip solid wall condition is imposed. The resulting velocity profile at the wall is visualized in Figure 9(b). 


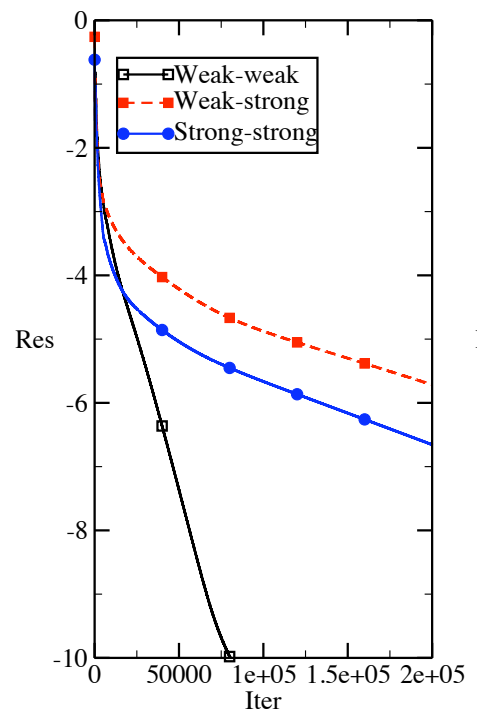

(a) Coarsest grid

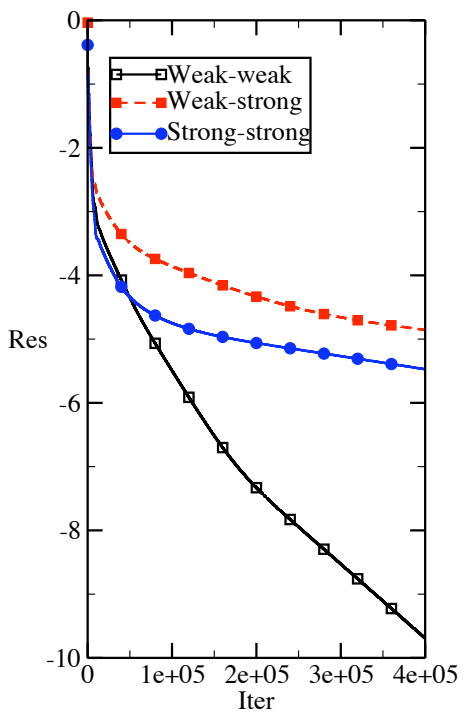

(b) Medium grid

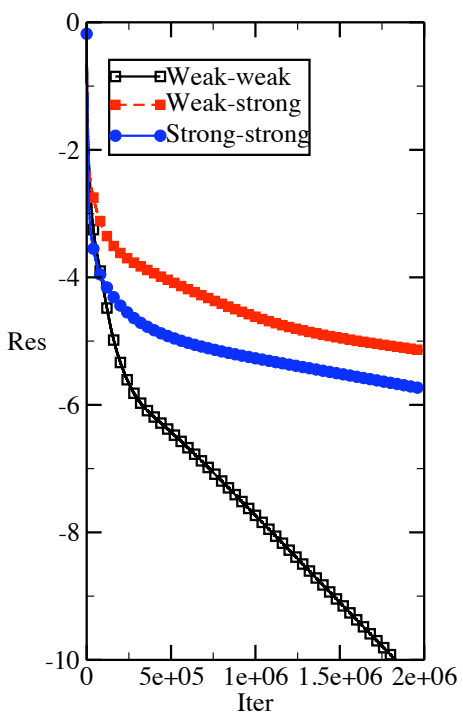

(c) Finest grid

Fig. 8. The convergence of the density residual. NACA0012 at Mach number $M=0.8$, Reynolds number $R e=500$ and $\alpha=0^{\circ}$ as the angle of attack.

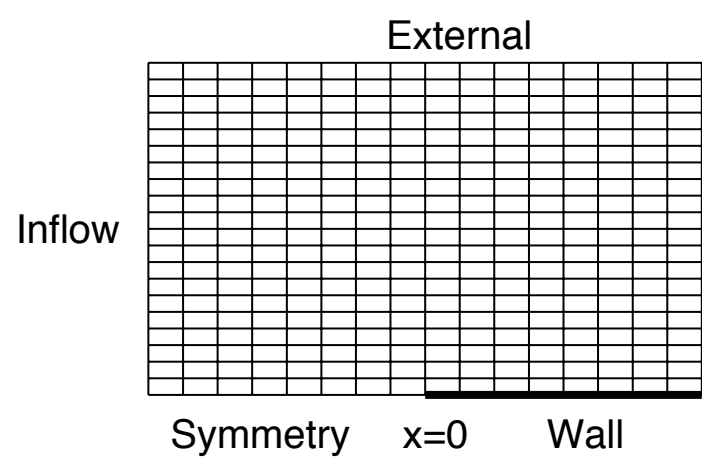

(a) Geometry

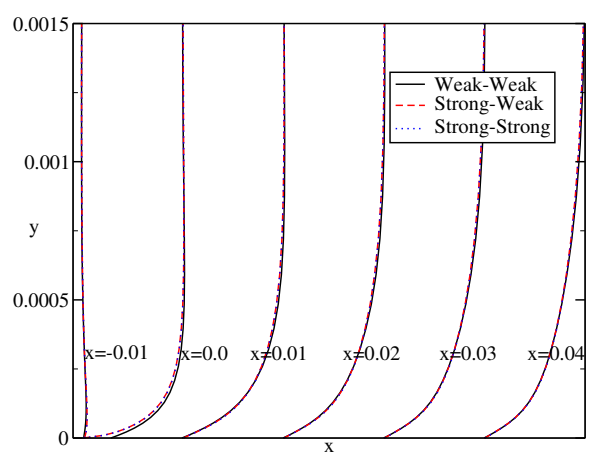

(b) Boundary layer

Fig. 9. The geometry of the flat plate and the flow at the solid wall.

The convergence of the density residual is shown in Figure 10. The weak scheme converges slightly faster than the mixed scheme, while the strong scheme does not converge at all. This behavior is also consistent with our previous linear analysis. Recall that for the model problem the strong scheme had an eigenvalue passing zero for the coarsest mesh (Figure 6(c)).

Next we investigate the influence of solid wall boundary procedures on the multigrid acceleration technique. We consider the same flow field (the flat plate) again. Now neither the mixed nor the strong boundary procedure converges, see Figure 11. This is probably due to the fact that eigenvalues close to or equal to zero (for our analogous linear problem) gives a non-decaying energy. Compare with Figure 6(d) where both the mixed and the strong discrete decay rates are magnitudes lower than the weak decay rate. 


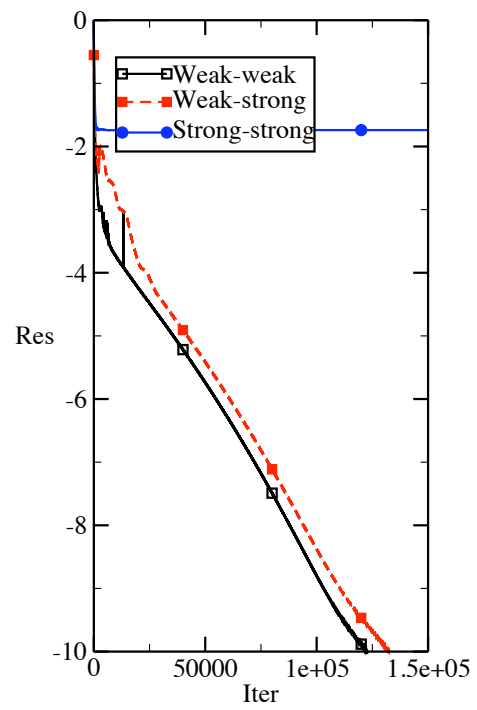

(a) Coarsest grid

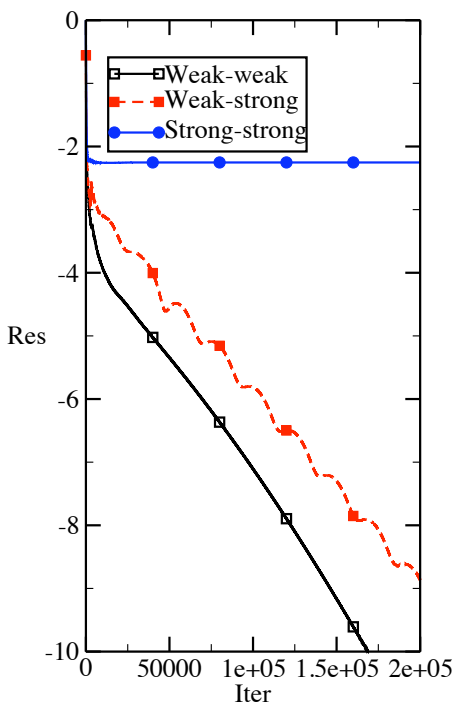

(b) Medium grid

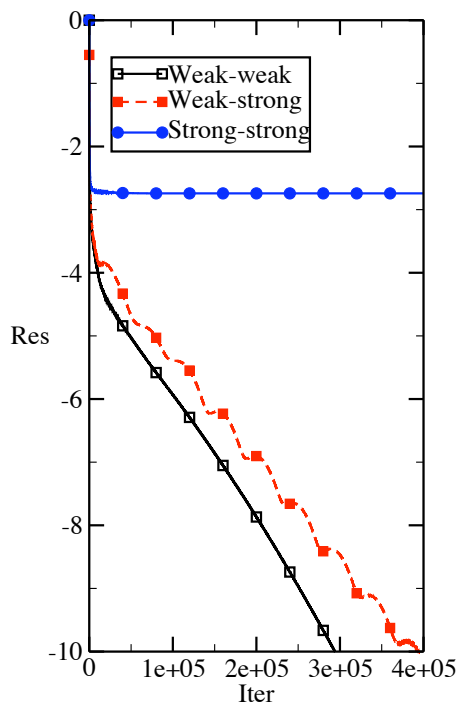

(c) Finest grid

Fig. 10. The convergence of the density residual. Flat plate at Mach number $M=0.07$ and Reynolds number per meter $R e / m=1.5 \cdot 10^{6}$.

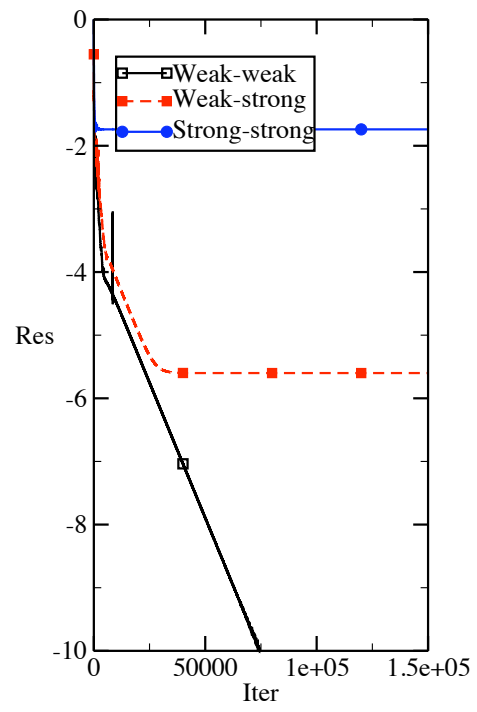

(a) Coarsest grid

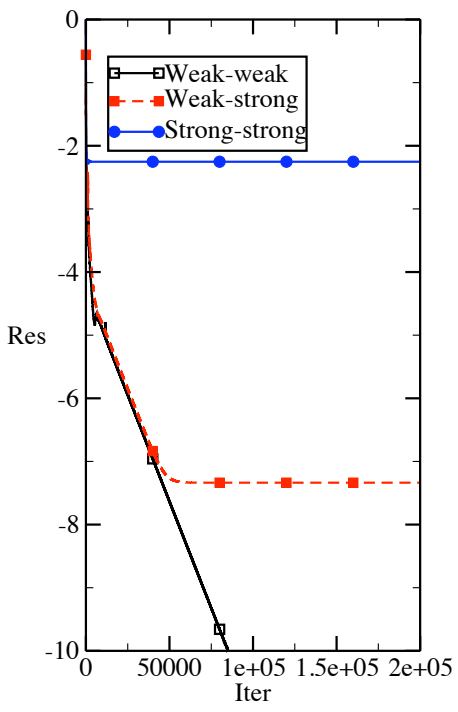

(b) Medium grid

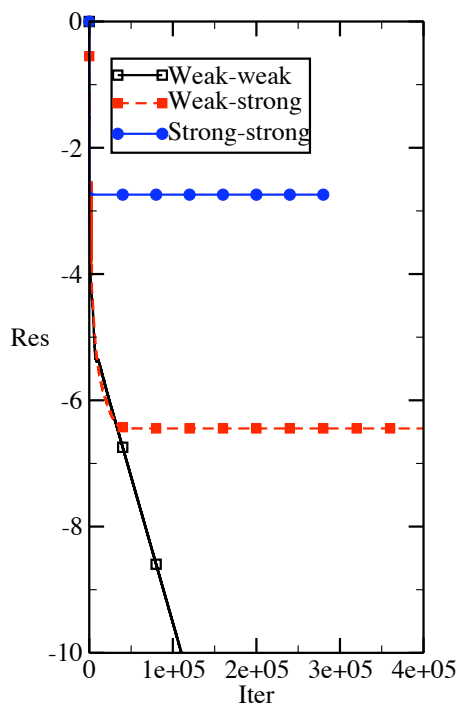

(c) Finest grid

Fig. 11. The convergence of the density residual. Flat plate, $M=0.07$, Reynolds number per meter $R e / m=1.5 \cdot 10^{6}$. Multigrid with three grid levels.

\section{Summary and conclusions}

We have studied how the choice of boundary procedure for solid walls affects the convergence to steady-state of the Navier-Stokes equations. Three kinds of boundary procedures were considered, namely a weak formulation, a strong formulation and a mixture of the weak and the strong formulation.

In the analysis part of the paper we considered the constant coefficient version 
of the Navier-Stokes equations with Fourier transformation in the $x$ direction. We proved stability for the weak and the mixed schemes using the energy method. No proof could be obtained for the strong formulation.

The discrete decay rate was obtained by computing the eigenvalues of the spatial operator for the three different boundary procedures. The discrete decay rate of the weak scheme was significantly larger lead to faster convergence to steady-state than the ones from the other two schemes. The difference was most pronounced for coarse meshes. For fine meshes the discrete decay rates converged to the continuous decay rate for all three boundary procedures.

Numerical calculations indicated that the linear results carries over to the fully nonlinear Navier-Stokes equations. Two flow cases, the steady flow around a NACA0012 airfoil and a flat plate, were considered. In all simulations the solution from the weak scheme converged fast, which was consistent with the linear analysis. The solutions obtained by the mixed and strong schemes either converged slower or, in some cases, did not converge at all.

\section{Acknowledgements}

This work has partly been carried out within the IDIHOM project which is supported by the European Commission under contract No. ACP0-GA-2010265780. Also acknowledged is the excellent working environment at CTR, The Center for Turbulence Research at Stanford University where the first two authors spent part of the time working on this paper.

\section{A A weak boundary procedure for the upper boundary}

For the upper boundary $(y=H)$ we use the Dirichlet boundary condition. On matrix form this is expressed as $L_{N} \hat{U}(1, t)+K_{N} \hat{U}(1, t)_{y}=0$, where $L_{N}=I_{4}$ and $K_{N}=0_{4}$. Inserting this into (22) yields $B T_{y=H}=0$, which does not add any energy growth. The boundary terms (corresponding to $(28)$ for $y=0$ ) is

$$
\begin{aligned}
B T_{N} & =\left[\begin{array}{c}
V_{N} \\
i \omega V_{N} \\
(\bar{D} V)_{N}
\end{array}\right]^{*}\left[\begin{array}{ccc}
-A_{2} & 0 & \varepsilon B_{22} \\
0 & -2 p \varepsilon B_{11} & -p \varepsilon\left(B_{12}+B_{21}\right) \\
\varepsilon B_{22}-p \varepsilon\left(B_{12}+B_{21}\right) & -2 p \varepsilon B_{22}
\end{array}\right]\left[\begin{array}{c}
V_{N} \\
i \omega V_{N} \\
(\bar{D} V)_{N}
\end{array}\right] \\
& +V^{*}\left(\bar{P} \Sigma_{N}+\Sigma_{N}^{*} \bar{P}\right) V .
\end{aligned}
$$


By using the penalty term $\Sigma_{N} V=\left(P^{-1} e_{N} \otimes \tau_{N}\right) V_{N}$ we obtain

$$
B T_{N}=[\begin{array}{c}
V_{N} \\
i \omega V_{N} \\
(\bar{D} V)_{N}
\end{array} \underbrace{\left[\begin{array}{ccc}
\tau_{N}+\tau_{N}^{*}-A_{2} & 0 & \varepsilon B_{22} \\
0 & -2 p_{N} \varepsilon B_{11} & -p_{N} \varepsilon\left(B_{12}+B_{21}\right) \\
\varepsilon B_{22} & -p_{N} \varepsilon\left(B_{12}+B_{21}\right) & -2 p_{N} \varepsilon B_{22}
\end{array}\right]}_{M_{N}}\left[\begin{array}{c}
V_{N} \\
i \omega V_{N} \\
(\bar{D} V)_{N}
\end{array}\right] .
$$

The matrix $M_{N}$, specified above, is made negative semi-definite by choosing the elements $\tau_{i j}$ of the $4 \times 4$ matrix $\tau_{N}$. Inserting the values $\tau_{12}=\tau_{21}=\tau_{14}=$ $\tau_{41}=\tau_{23}=\tau_{32}=\tau_{24}=\tau_{42}=0, \tau_{13}=\tau_{31}=\bar{c} /(2 \sqrt{\gamma})$ and $\tau_{34}=\tau_{43}=$ $\bar{c} \sqrt{(\gamma-1) / \gamma} / 2$ yields

$$
\begin{gathered}
B T_{N}=\left[\begin{array}{c}
\rho_{0} \\
T_{0} \\
i \omega T_{0} \\
(D T)_{0}
\end{array}\right]^{*} \underbrace{\left[\begin{array}{cccc}
\theta_{11} & 0 & 0 & 0 \\
0 & \theta_{22} & 0 & \theta_{24} \\
0 & 0 & \theta_{33} & 0 \\
0 & \theta_{42} & 0 & \theta_{44}
\end{array}\right]}_{M_{N}^{\theta}}\left[\begin{array}{c}
\rho_{0} \\
T_{0} \\
i \omega T_{0} \\
(D T)_{0}
\end{array}\right] \\
+\left[\begin{array}{c}
u_{0} \\
i \omega v_{0} \\
(D u)_{0}
\end{array}\right]^{\left[\begin{array}{ccc}
\psi_{11} & 0 & \psi_{13} \\
0 & \psi_{22} & \psi_{23} \\
\psi_{31} & \psi_{32} & \psi_{33}
\end{array}\right]}\left[\begin{array}{c}
u_{0} \\
i \omega v_{0} \\
(D u)_{0}
\end{array}\right]+\left[\begin{array}{c}
v_{0} \\
i \omega u_{0} \\
(D v)_{0}
\end{array}\right]_{M_{N}^{\psi}}^{\left[\begin{array}{ccc}
\varphi_{11} & 0 & \varphi_{13} \\
0 & \varphi_{22} & \varphi_{23} \\
\varphi_{31} & \varphi_{32} & \varphi_{33}
\end{array}\right]} \underbrace{\underbrace{\varphi}_{0}}_{M_{N}^{\varphi}}\left[\begin{array}{c}
v_{0} \\
i \omega u_{0} \\
(D v)_{0}
\end{array}\right]
\end{gathered}
$$

where

$$
\begin{array}{lll}
\psi_{11}=2 \tau_{22}-\bar{v} & \varphi_{11}=2 \tau_{33}-\bar{v} & \theta_{11}=2 \tau_{11}-\bar{v} \\
\psi_{13}=\varepsilon \frac{\mu}{\bar{\rho}} & \varphi_{13}=\varepsilon \frac{\lambda+2 \mu}{\bar{\rho}} & \theta_{22}=2 \tau_{44}-\bar{v} \\
\psi_{22}=-2 p_{N} \varepsilon \frac{\mu}{\bar{\rho}} & \varphi_{22}=-2 p_{N} \varepsilon \frac{\lambda+2 \mu}{\bar{\rho}} & \theta_{33}=-2 p_{N} \varepsilon \frac{\gamma \mu}{\operatorname{Pr} \bar{\rho}} \\
\psi_{23}=-p_{N} \varepsilon \frac{\lambda+\mu}{\bar{\rho}} & \varphi_{23}=-p_{N} \varepsilon \frac{\lambda+\mu}{\bar{\rho}} & \theta_{24}=\varepsilon \frac{\gamma \mu}{\operatorname{Pr} \bar{\rho}} \\
\psi_{33}=-2 p_{N} \varepsilon \frac{\mu}{\bar{\rho}} & \varphi_{33}=-2 p_{N} \varepsilon \frac{\lambda+2 \mu}{\bar{\rho}} & \theta_{44}=-2 p_{N} \varepsilon \frac{\gamma \mu}{\operatorname{Pr} \bar{\rho}} .
\end{array}
$$


The three sub-matrices $M_{N}^{\theta}, M_{N}^{\psi}$ and $M_{N}^{\varphi}$ are negative semi-definite if the following stability demands are fulfilled

$$
\begin{gathered}
\tau_{N}=\left[\begin{array}{cccc}
\tau_{11} & 0 & \bar{c} /(2 \sqrt{\gamma}) & 0 \\
0 & \tau_{22} & 0 & 0 \\
\bar{c} /(2 \sqrt{\gamma}) & 0 & \tau_{33} & \bar{c} \sqrt{\frac{\gamma-1}{\gamma}} / 2 \\
0 & 0 & \bar{c} \sqrt{\frac{\gamma-1}{\gamma}} / 2 & \tau_{44}
\end{array}\right], \quad \tau_{11} \leq \bar{v} / 2, \quad \tau_{44} \leq \bar{v} / 2-\frac{\varepsilon \gamma \mu}{4 \bar{\rho} P_{r} p_{N}} \\
\tau_{22} \leq \bar{v} / 2-\frac{\varepsilon \mu^{3}}{\bar{\rho}(\lambda+3 \mu)(\mu-\lambda) p_{N}} \quad \text { and } \quad \tau_{33} \leq \bar{v} / 2-\frac{\varepsilon(\lambda+2 \mu)^{3}}{\bar{\rho}(\lambda+3 \mu)(3 \lambda+5 \mu) p_{N}} .
\end{gathered}
$$

In the numerical simulations we used the limit values and $p_{N}=\Delta y / 2$.

\section{B Canceling terms for the strong scheme}

We have identified $\Sigma_{0}^{\text {strong }} V=\left(\tilde{C}_{0}+\tilde{C}_{1}+\tilde{C}_{2}\right) V$, where

$$
\begin{aligned}
& \tilde{C}_{0}=\left[\begin{array}{cccc|cccc}
0 & i \omega a & 0 & 0 & 0 & 0 & 0 & 0 \\
i \omega a & i \omega \bar{u}+\omega^{2} \varepsilon \varphi & 0 & i \omega b & 0 & 0 & 0 & 0 \\
0 & 0 & i \omega \bar{u}+\omega^{2} \varepsilon \psi & 0 & 0 & 0 & 0 & 0 \\
0 & i \omega b & 0 & i \omega \bar{u}+\omega^{2} \varepsilon \theta & 0 & -i \omega b & 0-i \omega \bar{u}-\omega^{2} \varepsilon \theta \\
\hline 0 & 0 & 0 & 0 & & \\
0 & 0 & 0 & 0 & & & \\
0 & 0 & 0 & 0 & & & \ddots
\end{array}\right],
\end{aligned}
$$

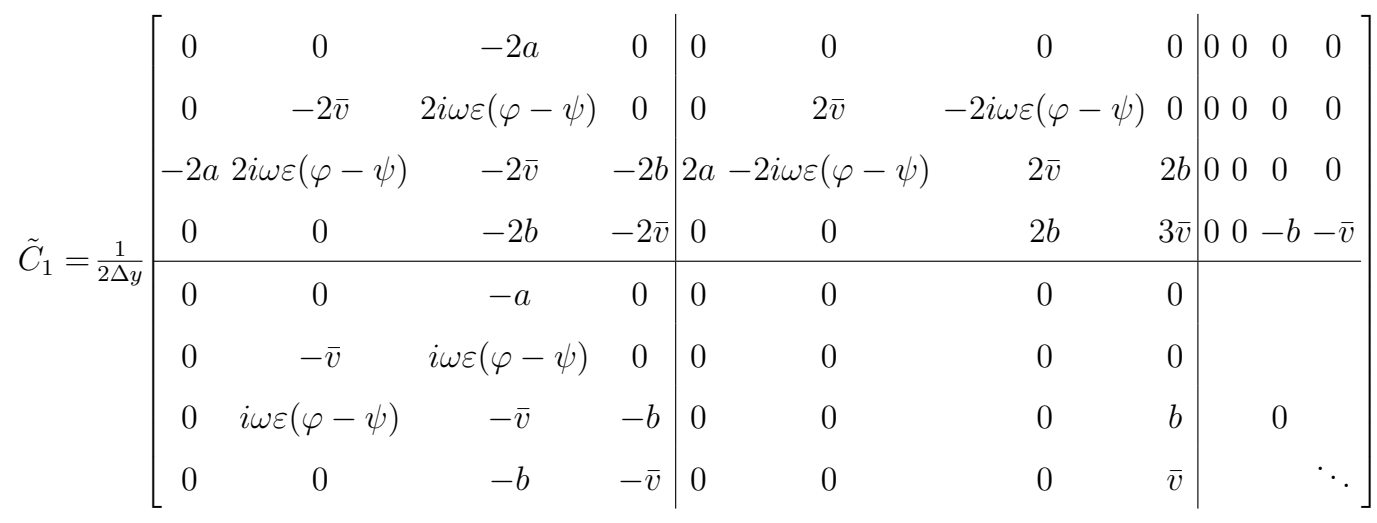


and

$$
\tilde{C}_{2}=\frac{-\varepsilon}{\Delta y^{2}}\left[\begin{array}{cccc|cccc|cccc}
0 & 0 & 0 & 0 & 0 & 0 & 0 & 0 & 0 & 0 & 0 & 0 \\
0 & 0 & 0 & 0 & 0 & 0 & 0 & 0 & 0 & 0 & 0 & 0 \\
0 & 0 & 0 & 0 & 0 & 0 & 0 & 0 & 0 & 0 & 0 & 0 \\
0 & 0 & 0 & 0 & 0 & 0 & 0 & \theta & 0 & 0 & 0 & -\theta \\
\hline 0 & 0 & 0 & 0 & 0 & 0 & 0 & 0 & & & \\
0 & \psi & 0 & 0 & 0 & 0 & 0 & 0 & & & \\
0 & 0 & \varphi & 0 & 0 & 0 & 0 & 0 & & 0 & \\
0 & 0 & 0 & \theta & 0 & 0 & 0 & -\theta & & & \ddots
\end{array}\right] .
$$

The abbreviations $a=\bar{c} / \sqrt{\gamma}, b=\bar{c} \sqrt{(\gamma-1) / \gamma}, \varphi=(\lambda+2 \mu) / \bar{\rho}, \psi=\mu / \bar{\rho}$ and $\theta=\gamma \mu /\left(P_{r} \bar{\rho}\right)$ have been used to shorten the expressions above.

\section{References}

[1] S. Abarbanel and D. Gottlieb. Optimal time splitting for two- and threedimensional Navier-Stokes equations with mixed derivatives. Journal of Computational Physics, 41:1-43, 1981.

[2] Q. Abbas and J. Nordström. Weak versus strong no-slip boundary conditions for the Navier-Stokes equations. Engineering Applications of Computational Fluid Mechanics, 4(1):29-38, 2010.

[3] J. Berg and J. Nordström. Stable Robin solid wall boundary conditions for the Navier-Stokes equations. Journal of Computational Physics, 230:7519-7532, 2011.

[4] M.H. Carpenter, J. Nordström, and D. Gottlieb. A stable and conservative interface treatment of arbitrary spatial accuracy. Journal of Computational Physics, 148:341-365, 1999.

[5] Bruno Costa, Wai Sun Don, David Gottlieb, and Radislav Sendersky. Twodimensional multi-domain hybrid spectral-WENO methods for conservation laws. Communications in Computational Physics, 1(3):548-574, Jun 2006.

[6] S. C. Dias and D. W. Zingg. A high-order parallel Newton-Krylov flow solver for the Euler equations. In 19th AIAA Computational Fluid Dynamics Conference, 2009 .

[7] Wai-Sun Don, David Gottlieb, and Jae-Hun Jung. A Weighted Multi-Domain Spectral Penalty Method with Inhomogeneous Grid for Supersonic Injective Cavity Flows. Communications in Computational Physics, 5(5):986-1011, May 2009 . 
[8] G. Efraimsson, J. Gong, M. Svärd, and J. Nordström. An investigation of the performance of a high-order accurate navier-stokes code. In Proc. ECCOMAS CFD Conference 2006, pages 11-. Tech. Univ. of Delft, 2006.

[9] P. Eliasson. Edge, a Navier-Stokes solver for unstructured grids. In Proceedings to Finite Volumes for Complex Applications III, pages 527-534, 2002.

[10] P. Eliasson, S. Eriksson, and J. Nordström. The influence of weak and strong solid wall boundary conditions on the convergence to steady state of the NavierStokes equations. AIAA Paper 2009-3551, 2009.

[11] P. Eliasson and P. Weinerfelt. Recent applications of the flow solver Edge. In Proceedings to 7th Asian CFD Conference, 2007.

[12] B. Engquist and B. Gustafsson. Steady state computations for wave propagation problems. Mathematics of Computations, 49:39-64, 1987.

[13] S. Eriksson, Q. Abbas, and J. Nordstrm. A stable and conservative method for locally adapting the design order of finite difference schemes. Journal of Computational Physics, 230(11):4216-4231, 2011. Cited By (since 1996): 1.

[14] S. Eriksson and J. Nordström. Analysis of the order of accuracy for nodecentered finite volume schemes. Applied Numerical Mathematics, 2009.

[15] J. Gong and J. Nordström. A stable and efficient hybrid scheme for viscous problems in complex geometries. Journal of Computational Physics, 226:12911309, 2007.

[16] D Gottlieb and JS Hesthaven. Spectral methods for hyperbolic problems. Journal of Computational and Applied Mathematics, 128(1-2, Sp. Iss. SI):83131, Mar 2001.

[17] B. Gustafsson, H.-O. Kreiss, and J. Oliger. Time Dependent Problems and Difference Methods. John Wiley \& Sons, Inc., 1995.

[18] JS Hesthaven and D Gottlieb. A stable penalty method for the compressible Navier-Stokes equations .1. Open boundary conditions. SIAM Journal on Scientific Computing, 17(3):579-612, May 1996.

[19] JS Hesthaven and T Warburton. Nodal high-order methods on unstructured grids - I. Time-domain solution of Maxwell's equations. Journal of Computational Physics, 181(1):186-221, Sep 2002.

[20] J. E. Hicken and D. W. Zingg. Superconvergent functional estimates from summation-by-parts finite-difference discretizations. SIAM Journal on Scientific Computing, 33(2):893-922, 2011.

[21] R. A. Horn and C. R. Johnson. Topics in Matrix Analysis. Cambridge University Press, 1991.

[22] X. Huan, J. E. Hicken, and D. W. Zingg. Interface and boundary schemes for high-order methods. In 19th AIAA Computational Fluid Dynamics Conference, 2009 . 
[23] A. Jameson, W. Schmidt, and E. Turkel. Numerical solution of the Euler equations by finite volume methods using Runge Kutta time stepping schemes. AIAA Paper 81-1259, 1981.

[24] J. E. Kozdon, E. M. Dunham, and J. Nordstrm. Interaction of waves with frictional interfaces using summation-by-parts difference operators: Weak enforcement of nonlinear boundary conditions. Journal of Scientific Computing, pages 1-27, 2011. Article in Press.

[25] H.-O. Kreiss and G. Scherer. Finite element and finite difference methods for hyperbolic partial differential equations, in: C. De Boor (Ed.), Mathematical Aspects of Finite Elements in Partial Differential Equation. Academic Press, New York, 1974.

[26] K. Mattsson. Boundary procedures for summation-by-parts operators. Journal of Scientific Computing, 18(1):133-153, 2003.

[27] K. Mattsson and J. Nordström. Summation by parts operators for finite difference approximations of second derivatives. Journal of Computational Physics, 199(2):503-540, 2004.

[28] D.J. Mavriplis. Accurate multigrid solution of the Euler equations on unstructured and adaptive meshes. AIAA Journal, 28(2), 1990.

[29] J. Nordström. The influence of open boundary conditions on the convergence to steady state for the Navier-Stokes equations. Journal of Computational Physics, 85:210-244, 1989.

[30] J. Nordström and M. H. Carpenter. Boundary and interface conditions for high order finite difference methods applied to the Euler and Navier-Stokes equations. Journal of Computational Physics, 148:621-645, 1999.

[31] J. Nordström and M. H. Carpenter. High-order finite difference methods, multidimensional linear problems and curvilinear coordinates. Journal of Computational Physics, 173:149-174, 2001.

[32] J. Nordström, K. Forsberg, C. Adamsson, and P. Eliasson. Finite volume methods, unstructured meshes and strict stability. Applied Numerical Mathematics, 45:453-473, 2003.

[33] J. Nordström and J. Gong. A stable and efficient hybrid method for aeroacoustic sound generation and propagation. Comptes Rendus Mecanique, 333:713-718, 2005 .

[34] J. Nordström and J. Gong. A stable hybrid method for hyperbolic problems. Journal of Computational Physics, 212:436-453, 2006.

[35] J. Nordström, J. Gong, E. van der Weide, and M. Svärd. A stable and conservative high order multi-block method for the compressible Navier-Stokes equations. Journal of Computational Physics, 228(24):9020-9035, 2009.

[36] J. Nordström and R. Gustafsson. High order finite difference approximations of electromagnetic wave propagation close to material discontinuities. Journal of Scientific Computing, 18(2):215-234, 2003. 
[37] J. Nordström, F. Ham, M. Shoeybi, E. van der Weide, M. Svärd, K. Mattsson, G. Iaccarino, and J. Gong. A hybrid method for unsteady fluid flow. Computers and Fluids, 38:875-882, 2009.

[38] J. Nordström and M. Svärd. Well-posed boundary conditions for the NavierStokes equations. SIAM Journal on Numerical Analysis, 43(3):1231-1255, 2005.

[39] M. Osusky, J. E. Hicken, and D. W. Zingg. A parallel Newton-Krylov-Schur flow solver for the Navier-Stokes equations using the sbp-sat approach. In 48 th $A I A A$ Aerospace Sciences Meeting Including the New Horizons Forum and Aerospace Exposition, 2010.

[40] W. E. Schiesser. The numerical method of lines : integration of partial differential equations. Academic Press, 1991.

[41] M. Shoeybi, M. Svrd, F. E. Ham, and P. Moin. An adaptive implicit-explicit scheme for the DNS and LES of compressible flows on unstructured grids. Journal of Computational Physics, 229(17):5944-5965, 2010.

[42] B. Strand. Summation by parts for finite difference approximation for $\mathrm{d} / \mathrm{dx}$. Journal of Computational Physics, 110(1):47-67, 1994.

[43] J.C. Strikwerda. Initial boundary value problems for incompletely parabolic systems. Commun. Pure Appl. Math., 9(3):797-822, 1977.

[44] M. Svärd, M.H. Carpenter, and J. Nordström. A stable high-order finite difference scheme for the compressible Navier-Stokes equations: far-field boundary conditions. Journal of Computational Physics, 225(1):1020-1038, 2007.

[45] M. Svärd, J. Gong, and J. Nordström. Stable artificial dissipation operators for finite volume schemes on unstructured grids. Applied Numerical Mathematics, 56(12):1481-1490, 2006.

[46] M. Svärd, J. Gong, and J. Nordström. An accuracy evaluation of unstructured node-centred finite volume methods. Applied Numerical Mathematics, 58:11421158, 2008.

[47] M. Svärd and J. Nordström. Stability of finite volume approximations for the Laplacian operator on quadrilateral and triangular grids. Applied Numerical Mathematics, 51:101-125, 2004.

[48] M. Svärd and J. Nordström. A stable high-order finite difference scheme for the compressible Navier-Stokes equations: No-slip wall boundary conditions. Journal of Computational Physics, 227(10):4805-4824, 2008.

[49] L. Zhou and H.F. Walker. Residual smoothing techniques for iterative methods. SIAM Journal on Scientific Computing, 15:297-312, 1994. 